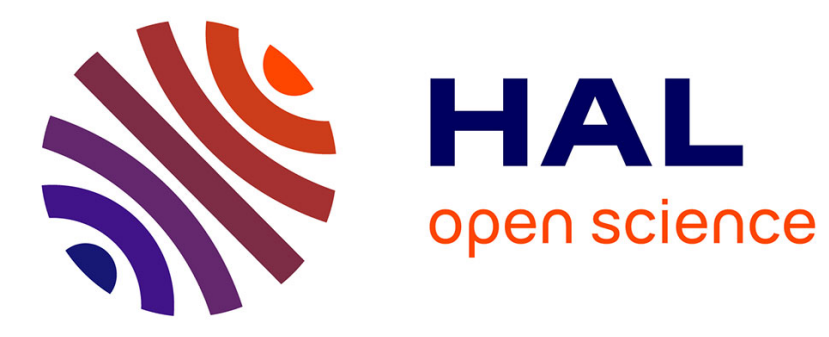

\title{
Mixing of a granular layer falling through a fluid
}

\author{
Michael Niebling, Eirik Grude Flekkøy, Knut J. Måløy, Renaud Toussaint
}

\section{To cite this version:}

Michael Niebling, Eirik Grude Flekkøy, Knut J. Måløy, Renaud Toussaint. Mixing of a granular layer falling through a fluid. Physical Review E: Statistical, Nonlinear, and Soft Matter Physics, 2010, 82 (1), 10.1103/PhysRevE.82.011301 . insu-02377764

\section{HAL Id: insu-02377764 https://hal-insu.archives-ouvertes.fr/insu-02377764}

Submitted on 24 Nov 2019

HAL is a multi-disciplinary open access archive for the deposit and dissemination of scientific research documents, whether they are published or not. The documents may come from teaching and research institutions in France or abroad, or from public or private research centers.
L'archive ouverte pluridisciplinaire HAL, est destinée au dépôt et à la diffusion de documents scientifiques de niveau recherche, publiés ou non, émanant des établissements d'enseignement et de recherche français ou étrangers, des laboratoires publics ou privés. 


\title{
Mixing of a granular layer falling through a fluid
}

\author{
Michael J. Niebling, ${ }^{1}$ Eirik G. Flekkøy, ${ }^{1}$ Knut Jørgen Måløy, ${ }^{1}$ and Renaud Toussaint ${ }^{2,3}$ \\ ${ }^{1}$ Department of Physics, University of Oslo, P.O. Box 1048, 0316 Oslo, Norway \\ ${ }^{2}$ Institut de Physique du Globe de Strasbourg, CNRS, 5 rue Descartes, 67084 Strasbourg Cedex, France \\ ${ }^{3}$ University of Strasbourg, 5 rue Descartes, 67084 Strasbourg Cedex, France \\ (Received 13 March 2009; revised manuscript received 18 May 2010; published 7 July 2010)
}

\begin{abstract}
We analyze the granular Rayleigh-Taylor instability of densely packed grains immersed in a compressible or an incompressible fluid using numerical simulations and two types of experiments. The simulations are based on a two-dimensional (2D) molecular dynamics model and the experiments have been carried out in systems of grains immersed in water/glycerol (incompressible fluid) and in air (compressible fluid). The variation of the interstitial fluid is shown to generate different dynamical patterns and mixing properties of the granular systems. The results have been quantified using $2 \mathrm{D}$ autocorrelation functions, the power spectrum of the velocity field and velocity field histograms. Excellent agreement is found between the numerical simulations and the experiments.
\end{abstract}

DOI: 10.1103/PhysRevE.82.011301

\section{INTRODUCTION}

The dynamics of particles immersed in fluids has been studied in a wide range of systems such as fluidized beds [1], sedimentation [2-4], mixing problems and granular flows. Many of the studied systems have important industrial applications as well as natural equivalents in a wide range of geological systems such as land slide, erosion, hydrofracturing [5], and avalanches [6].

The granular Rayleigh-Taylor instability has been studied recently using numerical as well as experimental methods for the case of air $[7,8]$ and for the case of grains falling in a highly viscous fluid [9-11]. Typically, experiments are conducted in closed rectangular Hele-Shaw cells where a dense granular layer falls through a gap filled with a fluid. In general, the falling layer separates into three different zones, in the top and bottom part of the cell sit compacted particles that barely move and in the space between these two zones, we have a region of moving particles of lower density perturbed by the spontaneous formation of fingerlike regions of higher particle density.

In contradistinction to previous works [7-11] where the focus has been on the evolution of the interface between the moving particle-zone and the compacted particles at the top of the cell, we shall here analyze in detail the bulk dynamics. In $[7,8]$ the granular Rayleigh-Taylor instability in air filled systems was studied and it was found that the finger formation differed qualitatively from systems filled with highly viscous fluids [9-11] and liquid-liquid systems. In the former case the finger formation was shown to coarsen in time, in contrast to observations in classical liquid-liquid systems and systems of grains immersed in highly viscous fluids, in these latter cases a single wavelength turned out to be dominating at all times.

Previously, the numerical modeling of the granular Rayleigh Taylor instability has been limited to systems with compressible interstitial fluids $[7,8]$. In the present work we extend the model to account for incompressible fluids. In addition we analyze the mixing dynamics over the whole cell and the sedimentation.

We compare the sedimentation and the particle dynamics for the case of air with the case of an incompressible liquid
PACS number(s): 81.05.Rm, 47.20.Ma, 47.11.-j, 45.70.Qj

by means of experiments and simulations. The particle dynamics are analyzed by velocity field histograms, twodimensional (2D) autocorrelation functions and the power spectrum of the velocity and density fields. Combining these methods we find excellent agreement between our numerical model and the experiments.

The velocity field of the experiments is extracted by the use of a correlation image velocimetry (CIV) technique. A similar technique was applied in studies of sedimentation of beads in silicone oil after the suspension was mixed with a small propeller $[3,4,12-15]$. In this paper we present a CIV method that reduces the noise of the displacement field by use of gray-level cross correlations as weights while averaging over several pixels. Our technique significantly improves the results obtained by standard procedures. The analysis of the velocity field gives quantitative interpretation of the different sedimentation patterns in the case of air compared to the case of the water/glycerol solution.

The paper is divided as follows. In the next section the experimental setup and experimental image analysis are described, followed in Sec. III by a description on the implementation of the numerical model. The results and the analysis are presented in Sec. IV. Physical interpretations and explanations of the mixing behavior are presented in Sec. V and the concluding remarks are given in Sec. VI.

\section{EXPERIMENTAL SETUP}

The experimental setup is shown in Fig. 1. We use a rectangular Hele-Shaw glass cell with the dimensions $5 \mathrm{~cm}$ in width by $8 \mathrm{~cm}$ in height and a plate spacing of $1 \mathrm{~mm}$. The plate spacing $h$ is achieved by a $1 \mathrm{~mm}$ thick silicone frame that ensures the cell to be tight toward three sides. Through the fourth side the beads, and when needed the beads in suspension with the fluid, are inserted. After the cell has been filled to the designated height of approximately $3 / 4$ with particles the fourth side is sealed with silicone paste leaving no air bubbles in the case of a fluid. In the case of air Dynoseed polystyrene beads with a mass density of $\rho_{m_{p}}=1.05 \mathrm{~g} / \mathrm{cm}^{3}$ and for water/glycerol glass beads with a mass density of $\rho_{m_{g}}=2.5 \mathrm{~g} / \mathrm{cm}^{3}$ are used. In both cases we sieved particles 


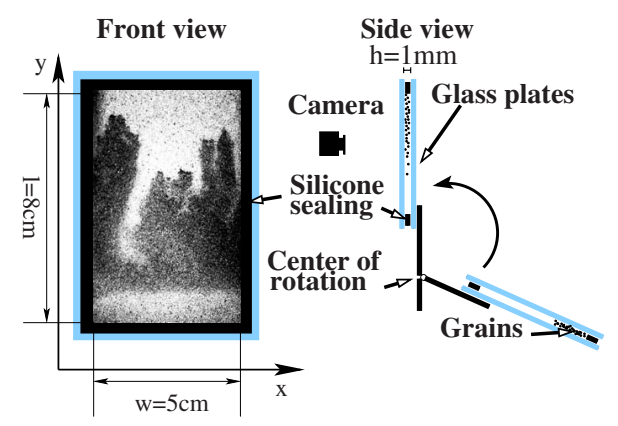

FIG. 1. (Color online) The experimental setup. The cell consists of two rectangular glass plates. Silicone sealing assures the cell to be leak proof. The front view shows a picture taken during an experiment with water/glycerol.

with an average diameter of $140 \pm 10 \% \mu \mathrm{m}$. This corresponds to a number of 180000 particles in the simulations.

After the preparation the cell is mounted by the use of clamps on a rotating bar. To ensure reproducibility, the sample was always prepared in the same way, flipping the cell a couple of times created a loose packing with an initially flat surface at the start of each experiment. Once the camera is running the cell is flipped upside down in about $0.2 \mathrm{~s}$ and pictures are taken at a rate of $1000 \mathrm{fps}$ (frames per second) in the case of air and $50 \mathrm{fps}$ in the case of water/ glycerol. For this purpose we use a high speed digital camera (Photron Fastcam-APX 120K) to record pictures with a resolution of $512 \times 1024$ pixels.

The rotation is stopped by a stopping bar that is softened by a piece of expending rubber. Despite the damping of the cell, the initial patterns are perturbated by the impact. In the analysis, we therefore disregard the early stage of the experiments.

The experiments with air are done under room conditions of $22^{\circ}$ in temperature and a relative humidity close to $30 \%$. Under this conditions the viscosity of air is $\mu_{a}$ $=0.000018 \mathrm{~Pa} \mathrm{~s}$. The water/glycerol solution has a mass concentration of $30 \%$ glycerol. At room conditions the viscosity was measured to be $\mu_{f}=0.00226 \mathrm{~Pa}$ s and the mass density of the solution was measured to be $\rho_{f}$ $=1.065 \mathrm{~g} / \mathrm{cm}^{3}$. It should be mentioned that a small amount of liquid soap was added to the water/glycerol solution in order to extract the small air bubbles sticking to the beads. However, it turned out that the soap lowered the interparticle friction as well as the friction between particles and the walls. Indeed, the soap approximately doubled the overall propagation speed of the unstable interface.

In all experiments, an amount of $10 \%$ of colored particles was randomly dispersed in the packing to increase the contrast for the numerical extraction of the velocity by the CIV technique. The colored particles were of the same density as the uncolored particles in the case of air and in the case of water/glycerol.

\section{Experimental picture analysis}

The subject of the analysis in this paper is the whole dynamic region of the granular Rayleigh-Taylor instability. A valuable quantity to study the dynamic region is the velocity field of the particles. In case of the simulations the velocities are computed at every time step and are directly accessible. For the experiments the velocity fields had to be extracted numerically from the pictures of the high speed camera by the means of a correlation image velocimetry (CIV) technique. For the CIV technique we use pictures of 255 graylevels with a resolution of $512 \times 1024$ pixels. The CIV technique is implemented in real space, according to classical techniques [16]. The displacement $d x, d y$ between two successive images at each point $x, y$ is determined by the maximization of the cross correlation of the gray map of squared zones centered at $x, y$ in the first image, and $x+d x, y+d y$ in the second one (Fig. 2). In addition to the classical treatment, we have developed an additional filtering method for the resulting velocity field, utilizing the fact that the obtained maximum cross correlation is a measure of the resemblance between the zones, and thus of the suitability of the CIV technique for the zone under consideration. To minimize the impact of artifacts due to aberrant zones where no resemblance is found with the following pictures, we convolve the velocity field obtained by a weight, that increases the better the cross correlation between the zones is. More specifically, for zones of interest of the size $2 i+1$ centered at $x_{0}, y_{0}$ and $x_{1}, y_{1}$, the cross correlation between gray-map $\mathrm{A}$ and $\mathrm{B}$ is defined as

$$
\begin{aligned}
& \left\langle A\left(x_{0}, y_{0}\right) B\left(x_{1}, y_{1}\right)\right\rangle_{i} \\
& \quad \equiv \sum_{m=-i}^{i} \sum_{n=-i}^{i} A\left(x_{0}+m, y_{0}+n\right) B\left(x_{1}+m, y_{1}+n\right)
\end{aligned}
$$

and

$$
\left\langle A\left(x_{0}, y_{0}\right)\right\rangle_{i} \equiv \sum_{m=-i}^{i} \sum_{n=-i}^{i} A\left(x_{0}+m, y_{0}+n\right) .
$$

For $s_{1}(x, y)$ and $s_{2}(x, y)$ the two gray-maps of two successive images, the normalized gray maps are defined as

$$
\begin{aligned}
& s_{1}^{\prime}(x, y)=s_{1}(x, y) / \sqrt{\left\langle s_{1}(x, y) s_{1}(x, y)\right\rangle_{i}}, \\
& s_{2}^{\prime}(x, y)=s_{2}(x, y) / \sqrt{\left\langle s_{2}(x, y) s_{2}(x, y)\right\rangle_{i}}
\end{aligned}
$$

and the cross-correlation between the two pictures as

$$
C(x, y, \Delta x, \Delta y)=\left\langle s_{1}^{\prime}(x, y) s_{2}^{\prime}(x+\Delta x, y+\Delta y)\right\rangle_{i} .
$$

The displacement $d x(x, y), d y(x, y)$ between two successive pictures for zones centered on $(x, y)$ is determined from maximizing $C(x, y, \Delta x, \Delta y)$ over $\Delta x, \Delta y$, i.e., such as:

$$
C(x, y, d x, d y)=\max _{\Delta x, \Delta y \leq l}[C(x, y, \Delta x, \Delta y)]=C_{\max }(x, y) .
$$

For this maximization, we used search values of $l$ $=6$ pixels maximum displacement for $\Delta x, \Delta y$, and sample squares of $7 \times 7$ with $i=3$ pixels. Next, the resulting displacement is convolved on running square windows of linear size $l$, with a weight, $w(x, y)=1 /\left[3-2 C_{\max }(x, y)\right]$ on each pixel, i.e., we determine a final average displacement 


\section{Picture at $\mathbf{t}_{0} \quad$ Picture at $\mathbf{t}_{0}+\mathbf{d t}$}

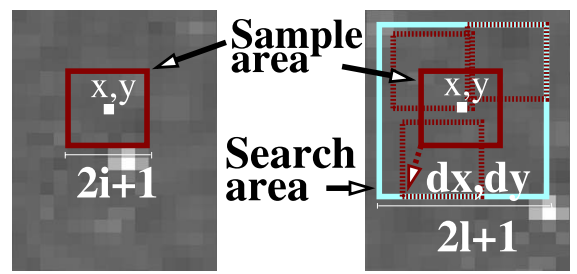

FIG. 2. (Color online) For the CIV technique the gray-level of two pictures in a time sequence is compared. In red (dark gray) a sample area is defined in the first picture. In the following picture it is then tried to find a similar area with the same gray-level distribution within the search area shown in blue (light gray).

$$
\begin{aligned}
d x_{f a}(x, y) & =\frac{\langle w(x, y) d x(x, y)\rangle_{i}}{\langle w(x, y)\rangle_{i}}, \\
d y_{f a}(x, y) & =\frac{\langle w(x, y) d y(x, y)\rangle_{i}}{\langle w(x, y)\rangle_{i}} .
\end{aligned}
$$

The resulting procedure is tested on simulated images, by comparing directly the resulting determined velocity field with the one calculated for the particles in the corresponding simulation .

We divide the average displacement by the time step between two pictures to get the averaged particle velocity components in $x$ and $y$ direction $u_{x}$ and $u_{y}$.

As stated before it is essential to have a good time resolution in the sequence of pictures. The size of the sample and search area has carefully been chosen according to the achieved resolution in our pictures. One pixel corresponds to $100 \mu \mathrm{m}$. To estimate how well our CIV technique is actually working we calculate the velocity field histogram from two pictures of the simulations one time with the CIV (left picture in Fig. 3) and another time by using the simulation data directly (right picture in Fig. 3). To make the direct simulation data comparable to the CIV data of the experiments we average the velocity of the single particles in the simulation with an equivalent procedure as in the experiments. In both pictures the velocity field is visualized on a square grid by

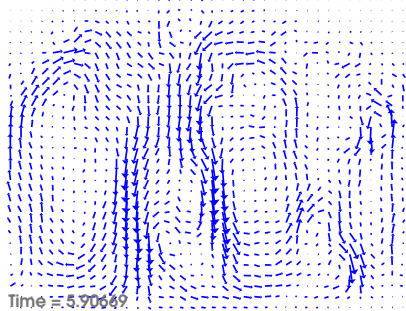

(a)

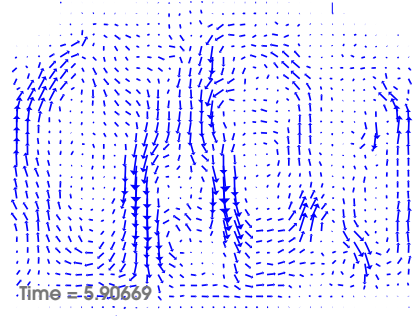

(b)
FIG. 3. (Color online) Test of the CIV technique: the left picture shows the velocity field derived directly from the simulation data in comparison with the right picture where the velocity field is obtained by the CIV technique from two pictures of the simulation. Both pictures show a section from the middle of the cell where most particles are moving.

vectors. The arrows represent the average of the velocity of the particles averaged over an area around the position of the arrow. To clarify the plot the size of the sample area was chosen to be $i=6, \quad(l=6)$ and was larger than the usual $i$ $=3$ pixels that we chose later for the analysis. In the plot the size of a vector represents the absolute value of the averaged velocity and the arrow points in direction of the averaged velocity vector. The velocity field in these two pictures only shows slight variations and the CIV technique reproduces the velocities well. For example we notice easily where fingers are falling down, the velocity is increased.

However when we analyze the experiments and simulations we will look at the velocity field histograms in $x$ and $y$ direction. Therefore it is necessary that the velocity field derived with the CIV technique leads to the same histograms as the one derived from the simulation data provided that we analyze the same picture. For this reason we compare the histograms derived by the CIV technique with the histograms derived from the simulations for the $x$-direction in graph 4(a) and for the $y$-direction in graph 4(b) in Fig. 4. In the histograms we divide $u_{x}$ and $u_{y}$ by the average absolute value of the velocity $u_{a}$. This compensates, that later the camera in the experiments records better the beads close to the glass plates which typically move slower. The velocity $u_{a}$ is defined as follows:
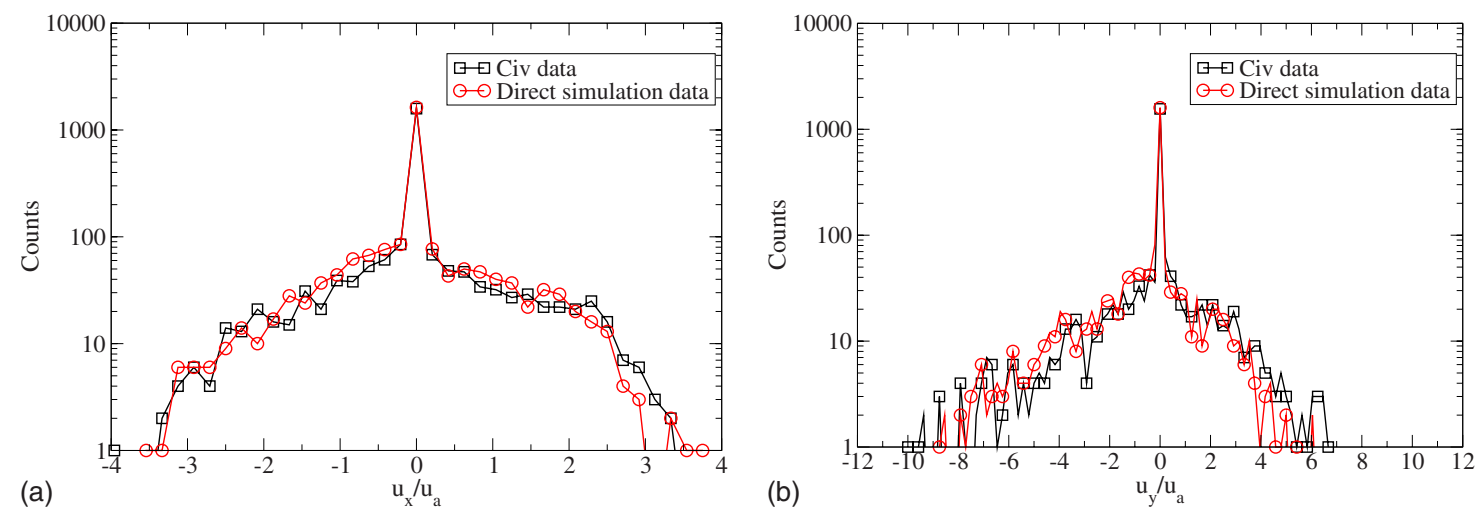

FIG. 4. (Color online) Histograms of the velocity field derived by the CIV technique and the velocity field derived directly from the simulation data. 


$$
u_{a}=\frac{1}{X Y} \sum_{i}^{X} \sum_{j}^{Y} \sqrt{u_{x}^{2}(i, j)+u_{y}^{2}(i, j)},
$$

where $X$ and $Y$ is the extend of the velocity field in $x$ and $y$ direction. In both figures good agreement between the two graphs is found showing that the velocity field extracted with the CIV analysis corresponds very well to the velocity field using the data directly from the simulations. By computing the deviation $\sigma_{u}$ between the velocity fields in Fig. 3 for areas where particles travel in distance between 0.9 and 3.2 of their diameter between two pictures, we found: $\sigma_{u}=\left\langle\left|\mathbf{u}_{\text {civ }}-\mathbf{u}_{\text {sim }}\right| / u_{\text {sim }}\right\rangle=0.19$. The average angle between these vectors of the two velocity fields is $\alpha$ $=\left\langle\left|\arccos \left[\left(\mathbf{u}_{\mathbf{c i v}} \cdot \mathbf{u}_{\text {sim }}\right) /\left(u_{\text {civ }} u_{\text {sim }}\right)\right]\right|\right\rangle=6.07^{\circ}$.

\section{THEORY AND SIMULATIONS}

The numerical model consists of two parts. One part is for the fluid using a continuum description derived from mass conservation and Darcy's law. The second part is for the particle motion using Newton's second law where the net force on a volume element balances its acceleration.

The model has been applied successfully in studies of instabilities of granular flows at low fluid Reynolds numbers $\operatorname{Re}=2 \rho_{f} u a / \mu$. Here $a$ is the particle radius, $\mu$ the viscosity and $\rho_{f}$ the fluid mass density. The theoretical derivation of the model is given in detail in [7,17-19]. Similar models were developed in [20-22] with a more detailed description of the fluid component.

In the following we will present the main features of the model and explain how to include incompressible fluids. We shall not consider the friction between the particles and restrict the model to two dimensions to keep the model computationally simple. However the friction of the granular flow with the glass plates was found to be essential and had to be included as will be shown in Sec. III D.

\section{A. Dynamics of the fluid-conservation of mass}

The fluid description for a compressible and an incompressible fluid is derived from mass conservation and Darcy's law for the fluid velocity through the porous media. A different point of view, starting from the Navier-Stokes equation at small term, and progressively averaging over volumes of various sizes, allows to obtain the same equations. For the sake of completion, this will be developed in the Appendix. It will also allow to obtain the form of various additional terms of small magnitude in the fluid stress, notably those related to the large scale velocity curvature. This approach shows the connection between the current model with the mixture theories sometimes used for fluidized beds or debris flows [23-26].

Here, we will directly use the Darcy law to express the seepage velocity of the fluid through the grains. The local permeability $\kappa$ is chosen by the Carman-Kozeny relation in the case of air and in the case of water/glycerol and can be written in terms of the local porosity $\phi$ and the local solid volume fraction $\rho_{s}=1-\phi$,

$$
\kappa=\frac{a^{2}}{9 K} \frac{\left(1-\rho_{s}\right)^{3}}{\rho_{s}^{2}}
$$

with $a$ the particle radius and $K=5$ an empirical constant valid for a packing of spherical beads [27].

For the mass conservation of the fluid we can write:

$$
\partial_{t}\left(\rho_{f} \phi\right)+\nabla \cdot\left(\rho_{f} \phi \mathbf{v}_{\mathbf{f}}\right)=0
$$

where $\mathbf{v}_{\mathbf{f}}$ is the velocity of the fluid. Similarly for the grains we get

$$
\partial_{t}(1-\phi)+\nabla \cdot[(1-\phi) \mathbf{u}]=0
$$

where $\mathbf{u}$ is the velocity of the grains. The velocity of the fluid $\mathbf{v}_{\mathbf{f}}$ has an advective term and a diffusive term. The advective term is caused by the motion of the grains and is equal to the velocity of the grains $\mathbf{u}$. The diffusive term is due to the gradient of the nonhydrostatic part of the pressure $P$ that results in a relative velocity of the fluid to the particles. $P$ corresponds to $P=P^{\prime}-\rho_{f} g y^{\prime}$, where $P^{\prime}$ is the pressure, $g$ the gravity constant, and $y^{\prime}$ the depth. With air as an interstitial fluid this diffusive term is described by a local Darcy flow $[28,29]$. The total velocity $\mathbf{v}_{\mathbf{f}}$ of the fluid is thus the sum of these two terms,

$$
\mathbf{v}_{\mathbf{f}}=\mathbf{u}-\frac{\kappa}{\phi \mu} \nabla P,
$$

where $\mu$ is the fluid viscosity. Inserting Eq. (12) in Eq. (10) and assuming that the fluid mass density $\rho_{f}$ is related to the pressure by the ideal gas equation $\rho_{f} \propto P$ we can write

$$
\partial_{t}(\phi P)+\nabla \cdot\left(\phi P\left[\mathbf{u}-\frac{\kappa}{\phi \mu} \nabla P\right]\right)=0 .
$$

$\partial_{t} \phi$ is eliminated in Eq. (13) by using Eq. (11) and by a few manipulations a diffusion equation is established for the nonhydrostatic part of the pressure $P$, the hydraulic head. This equation for the case of air includes fluid compressibility $[18,19]$

$$
\phi\left(\frac{\partial P}{\partial t}+\mathbf{u} \cdot \boldsymbol{\nabla} P\right)=\boldsymbol{\nabla} \cdot\left(P \frac{\kappa}{\mu} \boldsymbol{\nabla} P\right)-P \boldsymbol{\nabla} \cdot \mathbf{u} .
$$

In the case of a viscous incompressible fluid as water/ glycerol. The fluid mass density $\rho_{f}$ is not dependent on the pressure and the hydraulic head can be calculated by a Poisson equation, which we will derive briefly. By adding Eqs. (10) and (11) we get:

$$
\boldsymbol{\nabla} \cdot\left(\phi \mathbf{v}_{\mathbf{f}}\right)+\boldsymbol{\nabla} \cdot[(1-\phi) \mathbf{u}]=0 .
$$

A fluid flow between two plates results in a tangential stress $\sigma_{t}$ between the plates and the liquid. The origin of this stress term is discussed in more detail in the Appendix, Sec. VII.

The tangential stress between the plates and the fluid depends on the fluid viscosity. The increased viscosity in the case of the incompressible fluid makes this tangential stress potentially important. For this reason we will estimate the influence of this tangential stress with the side plates. If we solve Eq. (12) for the pressure gradient we add a contribution 


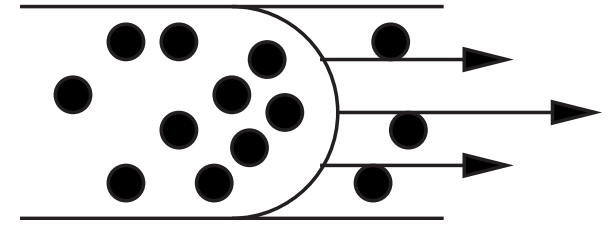

FIG. 5. Assuming a Poiseuille flow, a tangential stress between the plates and the particle fluid flow is deduced. This is a fluidmediated friction, present even without any interparticle contact or particle-plate contact.

to the pressure gradient due to the tangential stress $\sigma_{t}$ between the plates with plate spacing $h$ and the fluid, Eq. (12) gives

$$
\boldsymbol{\nabla} P=\frac{\sigma_{t}}{h}+\frac{\phi \mu}{\kappa}\left[\mathbf{u}-\mathbf{v}_{\mathbf{f}}\right] .
$$

The stress $\sigma_{t}$ between the fluid grain suspension and the glass plates depends on the fluid velocity $v_{f}$. The viscosity of this suspension $\mu_{g s}=\mu \phi^{-2.5}$ is obtained by the Einstein approximation [30] for spherical beads $[31,32]$ where the viscosity of the fluid $\mu$ is multiplied by $\phi^{-2.5}$. Based on an assumed parabolic flow profile as shown in Fig. 5 the tangential stress on the flow can be calculated,

$$
\sigma_{t}=-\frac{12 \mu \phi^{-2.5} \mathbf{v}_{\mathbf{f}}}{h} .
$$

Defining $\frac{1}{\kappa_{p}}=\frac{12 \phi^{-2.5}}{h^{2}}$ we can rewrite Eq. (16),

$$
\boldsymbol{\nabla} P=\frac{\phi \mu}{\kappa} \mathbf{u}-\left[\frac{\phi \mu}{\kappa}+\frac{\mu}{\kappa_{p}}\right] \mathbf{v}_{\mathbf{f}}
$$

we define $\frac{1}{\kappa^{\prime}}=\frac{1}{\kappa}+\frac{1}{\phi \kappa_{p}}$ and solve for the fluid velocity,

$$
\mathbf{v}_{\mathbf{f}}=\frac{\kappa^{\prime}}{\kappa} \mathbf{u}-\frac{\kappa^{\prime}}{\phi \mu} \nabla P .
$$

With Eq. (15) this results in a Poisson equation for the hydraulic head $P$,

$$
\boldsymbol{\nabla} \cdot\left(\frac{\kappa^{\prime}}{\mu} \nabla P\right)=\boldsymbol{\nabla} \cdot\left[\mathbf{u}\left(1-\phi+\frac{\kappa^{\prime}}{\kappa} \phi\right)\right],
$$

If we compare $f=\frac{\kappa}{\phi \kappa_{p}}$ we find for the maximum solid fraction $\rho_{s}=0.6 f=0.0057$ for the minimum solid fraction of $\rho_{s}$ $=0.15$ we find $f=0.063$. Under this conditions we can neglect the effect of the plates on the mass conservation part of the model and Eq. (18) reduces to Darcy's law, Eq. (12). For air, this reasoning leads to even smaller values of $f$, i.e., the correction due to the fluid coupling to the plates is even smaller, which justifies the use of Darcy's law. With incompressible fluid, Eq. (20) thus reduces to

$$
\boldsymbol{\nabla} \cdot\left(\frac{\kappa}{\mu} \nabla P\right)=\boldsymbol{\nabla} \cdot \mathbf{u}
$$

for water/glycerol.

Although we can neglect the stress $\sigma_{t}$ in this part in Sec. III B $\sigma_{t}$ contributes to the net force in Newton's second law. It has to be mentioned that the Carman-Kozeny relation is a

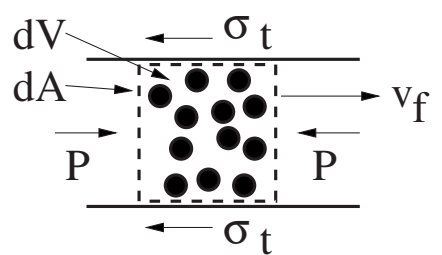

FIG. 6. Stress and pressure acting on the surface $d A$ of a unit volume $d V$.

relation where the solid volume fraction is considered as the volume fraction of a three-dimensional (3D) packing of spheres. In our simulation we consider the particles as cylinders with the base area $S_{a}=\pi a^{2}$ given by the particle radius and the height of the plate spacing $h$. The plate spacing of $1 \mathrm{~mm}$ allows approximately ten particles to be placed along the normal direction, i.e., our cylinder represents ten particles in height. For this reason we multiply the solid fraction of the cylinders by a factor of $2 / 3$ which is the solid fraction ratio between a randomly close packing of spheres to a randomly close packing of cylinders. The effect of this is to have the close packed solid fraction in 2D represent the close packed solid fraction in 3D.

Furthermore the Carman-Kozeny relation is not valid for solid fractions below 0.15 , therefore we use 0.15 as a cutoff in Eq. (9), any zone less dense is represented with a permeability corresponding to $\rho_{s}=0.15$. Such cutoff is also required numerically to avoid singularities during the solution of the differential Eq. (14) and (21) for the hydraulic head [18]. It effectively results in a code that rapidly homogenizes the pressure over zones with small solid fractions, which is physically sound.

\section{B. Dynamics of the particles-Newton's second law}

The movement of the particles under the effect of a fluid is subjected to buoyancy forces due to the mass density of the fluid $\rho_{f}$. To calculate the gravitational force we replace the mass density of the particles $\rho_{m}$ with the effective mass density $\rho_{e f f}=\rho_{m}-\rho_{f}$.

In a unit volume $d V$ with surface $d A$ the particle mass accounts to $\rho_{m} \rho_{s} d V$ and the fluid mass to $\rho_{f} \phi d V$ and we can write down the force equation in the following way:

$$
\begin{aligned}
\rho_{m} \rho_{s} d V \frac{d \mathbf{u}}{d t}+\rho_{f} \phi d V \frac{d \mathbf{v}_{\mathbf{f}}}{d t}= & \rho_{e f f} \rho_{s} d V \mathbf{g}+\sum \mathbf{F}_{\mathbf{I}}-\int P \mathbf{d} \mathbf{A} \\
& +\int \sigma_{t} d A+\mathbf{F}_{\gamma} .
\end{aligned}
$$

using $\mathbf{F}_{\mathbf{I}}$ as the interparticle force, $\mathbf{g}$ is the acceleration of the gravitation and $\mathbf{F}_{\gamma}$ accounts for the frictional forces and energy dissipation as will be specified further in Sec. III D. We also have to integrate the pressure and stress that is acting on the surface of this unit volume as shown in Fig. 6.

To simplify Eq. (22) we will justify in Sec. III C that the particles are small enough to move approximately with the fluid in a Lagrangian way. Under this conditions we can approximate the fluid velocity acceleration by the particle acceleration for the inertial term in the left hand side of Eq. (22), 


$$
\frac{d \mathbf{u}}{d t} \approx \frac{d \mathbf{v}_{\mathbf{f}}}{d t}
$$

and Eq. (22) results in

$$
\begin{aligned}
{\left[\rho_{m} \rho_{s} d V+\rho_{f} \phi d V\right] \frac{d \mathbf{u}}{d t}=} & \rho_{e f f} \rho_{s} d V \mathbf{g}+\sum \mathbf{F}_{\mathbf{I}}-\int \boldsymbol{\nabla} P d V \\
& +\int \sigma_{t} d A+\mathbf{F}_{\gamma} .
\end{aligned}
$$

Note that this approximation only affects the description of the fluid inertia, and not the drag force from the fluid on the particles. For a single particle of mass $m=\rho_{m} \pi a^{2} h$, volume $V_{a}=\pi a^{2} h$ and plate spacing $h$ we can define the number density $\rho_{n}=\rho_{s} \rho_{m} / m$ and use in Eq. (24) the unit volume $d V$ $=1 / \rho_{n}$,

$$
\left[m+\rho_{f} \frac{\phi}{\rho_{n}}\right] \frac{d v_{p}}{d t}=\rho_{e f f} V_{a} \mathbf{g}+\mathbf{F}_{\mathbf{I}}-\frac{\boldsymbol{\nabla} P}{\rho_{n}}+\frac{\sigma_{t} S_{a}}{\rho_{s}}+\mathbf{F}_{\gamma_{\mathbf{p}}} .
$$

This is the force equation for each single particle with the velocity $v_{p}$. The mass of the fluid appears in the first term of this equation and is simply added to the mass of a particle. The amount of mass from the fluid that we add to the mass of each particle depends on the local porosity divided by the number density. This allows to incorporate the fluid inertia to the model, by changing the particle mass into a fluid-coated particle mass.

The interparticle force $\mathbf{F}_{\mathbf{I}}$ is set to be a linear force with a spring constant $k$. The strength of this force is proportional to the overlap of the particles $d$ and the direction of this force is given by the unit vector $\mathbf{n}_{\mathbf{d}}$ which points from the center of the respective bead to the contact point. We choose the spring constant $k$ strong enough, so that our particles are approximately hard spheres. That means that the overlap of the particles that occur during the simulations are a negligible fraction of their distance.

$$
\mathbf{F}_{\mathbf{I}}(d)=-k d \mathbf{n}_{\mathbf{d}} .
$$

The particle propagation is modeled by the velocity verlet scheme [33].

\section{Fluid velocity relative to the particles}

To justify our simplification in Eq. (22) we compare the relative velocity between the grains and the fluid $v_{d}$ $=\frac{\kappa}{\phi \mu} \boldsymbol{\nabla} P$ with the absolute velocity of the grains $\mathbf{u}$. Moreover, the acceleration of the fluid relative to the particles is compared to the absolute acceleration of the particles. For this test we study two systems. First is the system that we use for the comparison with the experiments. In this system the fluid mass is added to the grains and the friction with the plates due to the tangential stress $\sigma_{t}$ is taken into account. Furthermore we test a second system where the fluid mass density $\rho_{f}$ in Eq. (22) is set to zero and the friction due to the tangential stress $\sigma_{t}$ is not taken into account. Such a system has a larger mass density contrast between the fluid and the particles. Consequently the difference between the accelera-
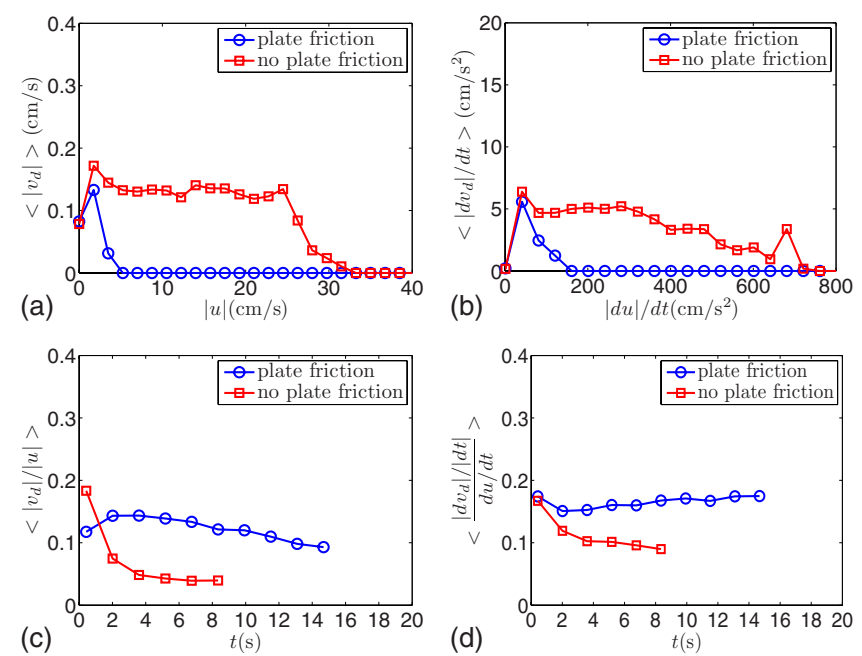

(b)

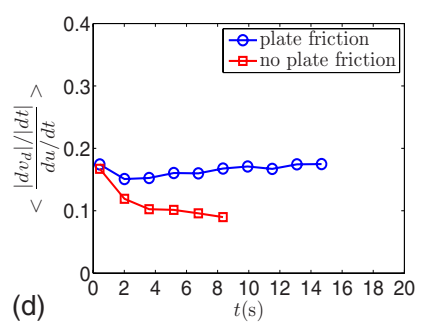

FIG. 7. (Color online) Comparison of the relative to the grains acceleration and relative to the grains velocity of the fluid to the absolute acceleration and absolute velocity of the grains. A system with plate friction due to a Poiseuille flow and added fluid mass to the particles (blue curves with circles) is compared to a system without friction due to a Poiseuille flow and without added fluid mass (red curves with squares).

tion of the grains and the fluid must be larger than in our case with $\rho_{f}=1.065 \mathrm{~g} / \mathrm{cm}^{3}$ where the mass density of the fluid is closer to the mass density of the grains. Obviously, for smaller mass densities of the particles relative to the fluid density, the particles are dragged more easily along with the fluid. The results of this comparison are shown in Fig. 7 in the blue curves (marked with circles) for the first system and for the red curves (marked with squares) for the second system. In Figs. 7(a) and 7(b) the plots are averaged in time over the whole simulations. These plots show for the two tested systems that the relative fluid acceleration is small compared to the total fluid acceleration and also that the relative fluid velocity is small compared to the absolute fluid velocity. It should be emphasized that the relative fluid velocity becomes a smaller and smaller fraction of the absolute fluid velocity the higher the absolute fluid velocity gets in Fig. 7(a). This also holds for the acceleration as shown in Fig. 7(b). This means that the more inertia is connected to the grains and fluid flow, the better our approximation in Eq. (23) holds. At low accelerations and granular velocities this approximation becomes less accurate but at the same time the contribution in Eq. (24) due to the acceleration: $\left[\rho_{m} \rho_{s} d V+\rho_{f} \phi V\right] \frac{d \mathbf{u}}{d t}$ and due to the velocity: $\sigma_{t}$ are getting less important.

The evolution in time of the fraction between the relative fluid velocity and the absolute grain velocity is shown in Fig. $7(\mathrm{c})$ and between the relative fluid acceleration and the absolute grain acceleration is shown in Fig. 7(d). The fraction of the acceleration was averaged over all absolute grain accelerations larger than $\frac{d \mathbf{u}}{d t} \geq 20 \mathrm{~cm} / \mathrm{s}^{2}$ as a cutoff which is $2 \%$ of the gravitational acceleration.

For the velocity we only averaged over values where the absolute grain velocity was larger than $\mathbf{u} \geq 1 \mathrm{~cm} / \mathrm{s}$, at this velocity the tangential stress arising from coupling with the plates $\sigma_{t}$ has a significant contribution of $18 \%$ of the gravi- 


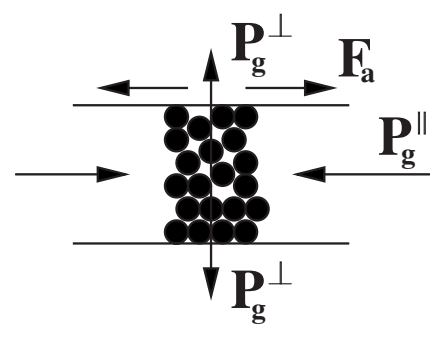

FIG. 8. The Janssen effect: the in-plane $P_{g}^{\|}$stress is being deflected by the particles resulting in a normal stress $P_{g}^{\perp}$ on the plates.

tational acceleration. The check of the velocity and the acceleration differences between the grains and the fluid reveals that the fluid is largely accelerated in the same way as the grains and the difference in acceleration is less than $20 \%$ even though we examined a system with a larger density contrast between the grain and the fluid mass density than what we have in our water/glycerol solution. In the plots 8(a) and 8 (b) we notice that the plate friction reduces the absolute grain velocity significantly. In the blue graph (marked with circles) in Fig. 7(a), maximum grain velocities are $5 \mathrm{~cm} / \mathrm{s}$ which makes inertia effects less important than in the system without plate friction.

\section{Solid friction forces}

In addition to the friction between the fluid and the plates we also consider the friction between the glass plates and the particles according to the normal stress $P_{g}^{\perp}$ in form of a Coulomb friction model into account. It is most active in the top section and the sediments where we find not moving compacted particles. This mechanism prevents the top section of compacted particles from getting unstable and falling toward one side of the cell as a whole due to a small initial perturbation in the thickness of this layer of beads that inflates rapidly without the friction between the grains and the plates as a opposing force. Here the normal stress of the particles onto the plates is derived by assuming a local Janssen hypothesis (see Fig. 8). The average in-plane stress tensor $\left\langle\sigma_{i j}^{\|}\right\rangle$exerted over a particle with index $a$ in contact with a set $C_{a}$ of particles $b$ is obtained as $[17,34]$

$$
\left\langle\sigma_{i j}^{\|}\right\rangle=\frac{1}{V_{a}} \sum_{b \in C_{a}} x_{i}^{b} f_{j}^{b}
$$

$x^{b}$ is the position of the contact with grain $b, f^{b}$ the contact force between the particle $a$ and $b$ and $i, j$ are Cartesian indices. $V_{a}=\pi a^{2} h$ is the particles volume which is described by a cylinder for a plate spacing $h$. Equation (27) can be proven in the following way. Using the integral theorem of Gauss and replacing the sum over the surface of one particle in Eq. (27) by the surface integral over the surface of the particle and applying the Einstein sum convention we get

$$
\begin{aligned}
\oint x_{i} \sigma_{k j}^{\|} n_{k} d A & =\int_{V_{a}} \nabla_{k}\left(x_{i} \sigma_{k j}^{\|}\right) d V \\
& =\int_{V_{a}}\left[\left(\nabla_{k} x_{i}\right) \sigma_{k j}^{\|}+\left(\nabla_{k} \sigma_{k j}^{\|}\right) x_{i}\right] d V .
\end{aligned}
$$

In the top section and the sediments where particles are com- pressed and the Janssen effect is primarily active particles are in a quasistatic state. In this case inertial terms are negligible in front of contact forces, so that internal force balance holds: $\nabla_{k} \sigma_{k j}^{\|}=0$ [35]. In Eq. (28) we can write

$$
\oint x_{i} \sigma_{k j}^{\|} n_{k} d A=\int_{V_{a}} \delta_{k i} \sigma_{k j}^{\|} d V=V_{a}\left\langle\sigma_{i j}^{\|}\right\rangle
$$

and proof Eq. (27). Taking the trace of the stress tensor in Eq. (27) we get the magnitude of the in-plane stress as: $P_{a}^{\|}$ $=-\left(\left\langle\sigma_{11}^{\|}\right\rangle+\left\langle\sigma_{22}^{\pi}\right\rangle\right) / 2$. According to the Janssen hypothesis the normal stress is proportional to the in-plane stress by a factor $\lambda$. With a Coulomb friction model we get that the frictional force $F_{a}$ per particle is proportional to the normal stress by a friction coefficient $\gamma$. The factor 2 accounts for the glass plates on each side of the particle.

$$
F_{a} \leq 2 \gamma S_{a} P_{g}^{\perp}=2 \gamma \lambda S_{a} P_{g}^{\|}
$$

This results in finding a value for $\gamma \cdot \lambda$. To get an estimate of $\gamma$ we performed some basic experiments. In these experiments a layer of beads was glued to the underpart of a weight, which is placed on a glass plate. Lifting one side of the glass plate we increase the angle of inclination $\alpha$ gradually and measure the maximum angle $\alpha_{s}$ before the weight with the beads underneath starts to slide. We repeated this experiment under water and got in both cases for the constant $\gamma=\tan \left(\alpha_{s}\right) \approx 0.22$. For $\lambda$ we know that it has to be smaller than 1 since the normal stress resulting from the Janssen effect can only be as large as the in-plane stress. With this idea for the range of the values we chose the constant $\gamma \lambda=0.07$ for the case of water/glycerol and $\gamma \lambda=0.042$ in the case of air. These values were fine tuned by fitting the propagation speed of the interface between the top layer and the dynamic zone in the simulation to the experimental results. We also tried $\gamma \lambda=0.07$ for the case of air with the result, that the simulations differed slightly more from the experiments. However the characteristics of the velocity field distributions remained unchanged.

We also include energy dissipation if particles collide [8]. This is done by introducing a viscous force negative proportional to the relative velocity of the particles $\mathbf{v}_{\mathbf{r}}$ projected on the unit vector $\mathbf{n}_{\mathbf{d}}$, which points from the center of one particle to the contact point. The direction of this force is given by the unit vector $\mathbf{n}_{\mathbf{d}}$ :

$$
\mathbf{F}_{\mathbf{d}}=-\gamma_{d}\left(\mathbf{v}_{\mathbf{r}} \cdot \mathbf{n}_{\mathbf{d}}\right) \mathbf{n}_{\mathbf{d}} .
$$

For the simulations it is important to have energy dissipation of more than $20 \%$ for each collision. From energy dissipation of $20 \%$ upward the evolution of the simulations does not change noticeable. For energy dissipation under $20 \%$ and less, increasing perturbations are observed as shown in [8]. The factor $\gamma_{d}$ was chosen to simulate a energy dissipation of more than $20 \%$ of the energy for each collision with a restitution coefficient of 0.55 . These two mechanisms result in the force $F_{\gamma}$ in Eq. (25). 


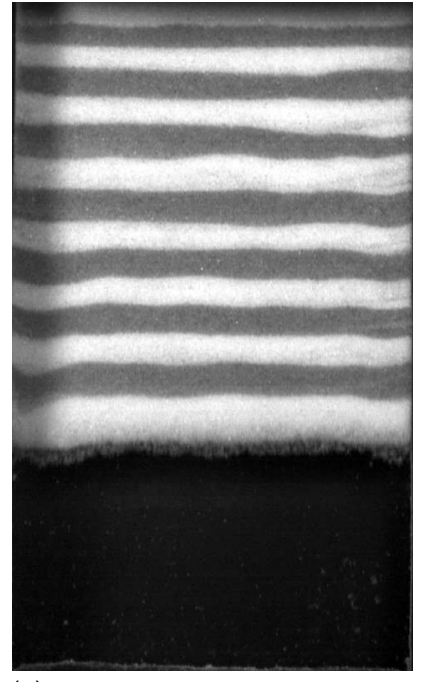

(a)

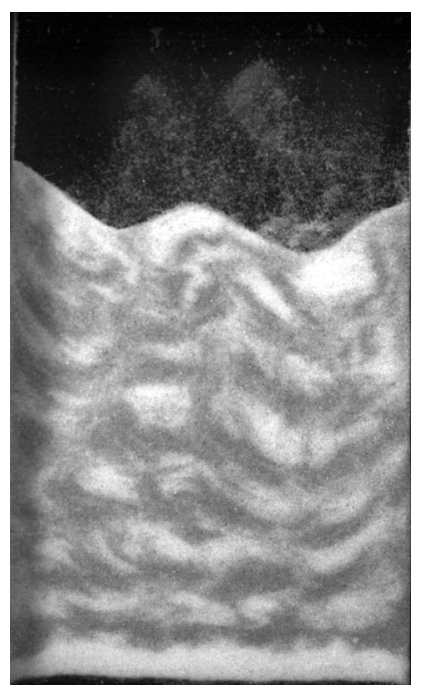

(c)

FIG. 9. Experiments showing different sedimentation patterns for the cases of air and water/glycerol.

\section{RESULTS AND COMPARISON BETWEEN EXPERIMENTS AND SIMULATIONS}

To illustrate the difference between experiments with water/glycerol and experiments with air we performed two experiments shown in Fig. 9 and two simulations shown in Fig. 10, for the two studied cases of grains with air and grains with water/glycerol.

At the start of the experiments and simulations [pictures (a) and (b) of Figs. 9 and 10] we have aligned particles with different colors at the top of a cell.

By comparison of the resulting sedimentation patterns after the experiments and simulations [pictures (c) and (d) in Figs. 9 and 10] are finished we already get a clear distinction between the two cases of air and water/glycerol. While in the case of air the structure of the initial layers of colored particles survives, we observe a complete mixing in the case of water/glycerol, apart from two chunks remaining lowly perturbed, in the case of the simulations. Such chunks have also

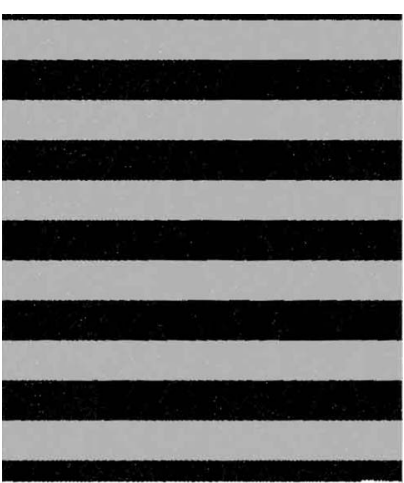

(a)

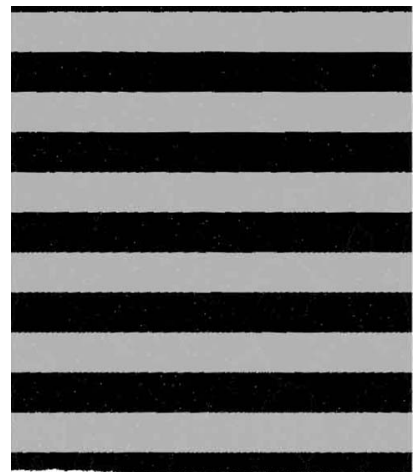

(b) (c)

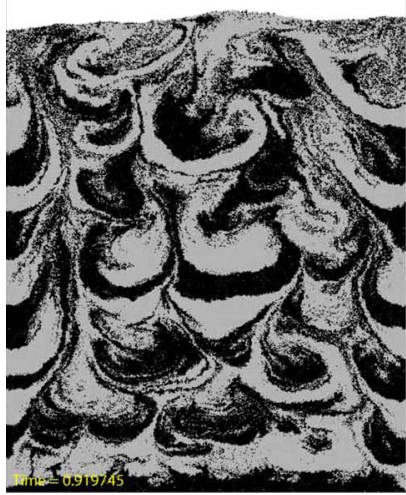

(d)

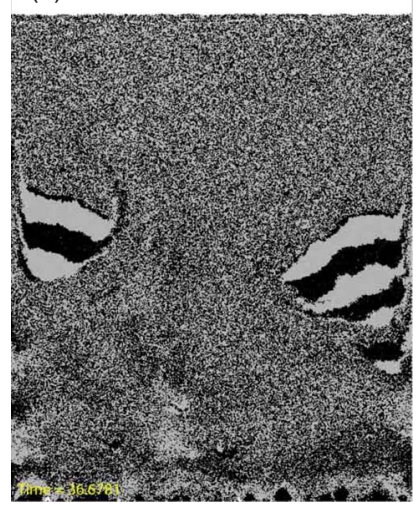

FIG. 10. (Color online) Simulations showing different sedimentation patterns for the cases of air and water/glycerol.

been observed in following experiments. The reason why none of these lowly perturbated areas occurred in the samples confining particles deposited in layers, lies to some extent in the preparation procedure of these samples, which always were the first in a series of experiments. Creating a sample with layers meant to move the cell from where it was filled to the mounting where it was exposed to additional stress from clamps. This resulted in a slightly higher compaction of the cell which increased the normal stress on the particles that resulted in a higher friction of the particles with the glass plates. For analyzing purpose only following experiments were used where the sample remained in the mounting under constant stress from the clamps.

\section{Analysis}

In our experiments we chose the viscosity of the water/ glycerol mixture in a way that the Reynolds number with respect to the plate spacing $\mathrm{Re}_{h}$ during the experiments was sufficient small to assume laminar flow. Typically the Reynolds number was less than $\mathrm{Re}_{h} \leq 5$ during the experiments with water/glycerol, and less than $\operatorname{Re}_{h} \leq 10$ in the case of air. 


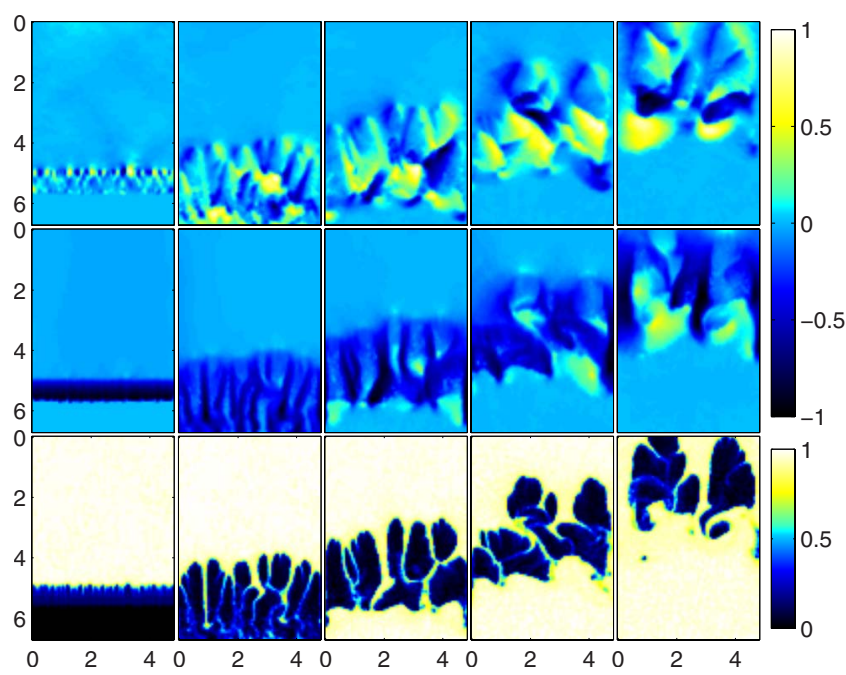

(a)

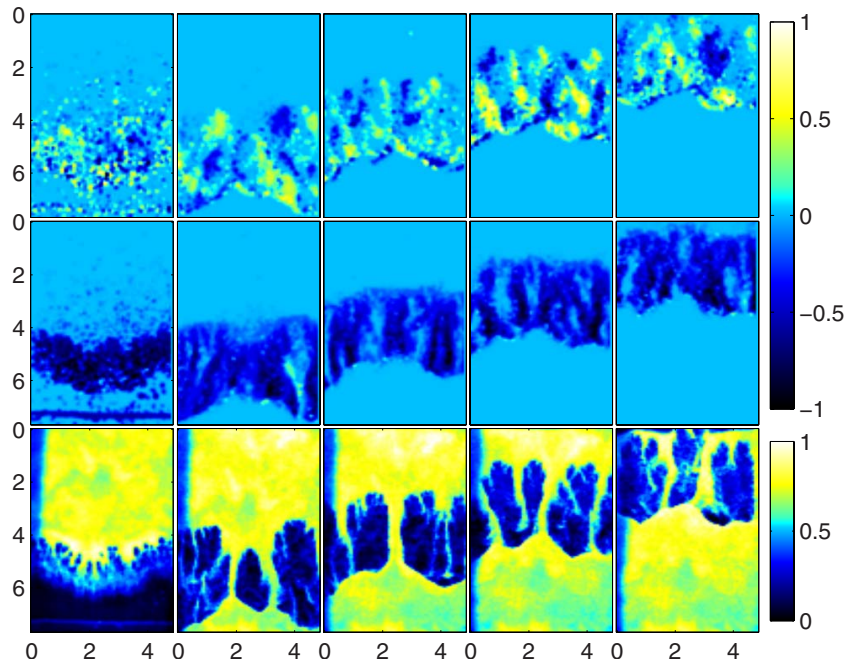

(b)

FIG. 11. (Color online) The three rows in figure (a) and (b) show the time evolution of the simulations (a) and experiments (b) with air from left to right at times $t=0.05 \mathrm{~s}, t=0.19 \mathrm{~s}, t=0.36 \mathrm{~s}$, $t=0.46 \mathrm{~s}$, and $t=0.6 \mathrm{~s}$. From top to bottom the rows illustrate the $u_{x} / \max \left(\left|u_{x}\right|\right)$ component and the $u_{y} / \max \left(\left|u_{y}\right|\right)$ component of the grain velocity vector field and the bottom row shows the density field $\rho_{d} / \max \left(\rho_{d}\right)$. All quantities have been normalized by its maximum absolute value.

In the sequence of pictures in Fig. 11 simulations are compared with experiments and the evolution of the granular velocity field $\mathbf{u}$ and the density field $\rho_{d}$ is shown for the case of air. From left to right time is progressing in equal steps from 0.05 to $0.6 \mathrm{~s}$ after the cell has been flipped. In the sequence of pictures in Fig. 12 the evolution of the simulations and experiments for water/glycerol is shown in the same way, except that here the time runs from 0.8 to $16.8 \mathrm{~s}$ after the cell has been flipped.

In this Figs. 11 and 12 we show color maps in the three rows from top to bottom of the $x$ component, the $y$ component of the velocity field $\mathbf{u}$ and the particle density field $\rho_{d}$. The velocity fields are calculated after the procedure described in Sec. II. The density field is in the case of the

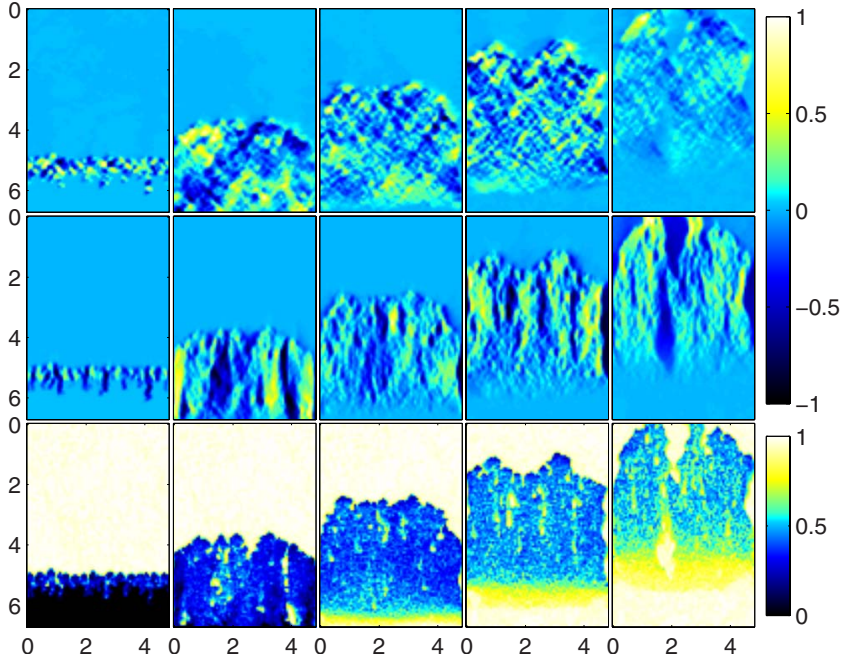

(a)

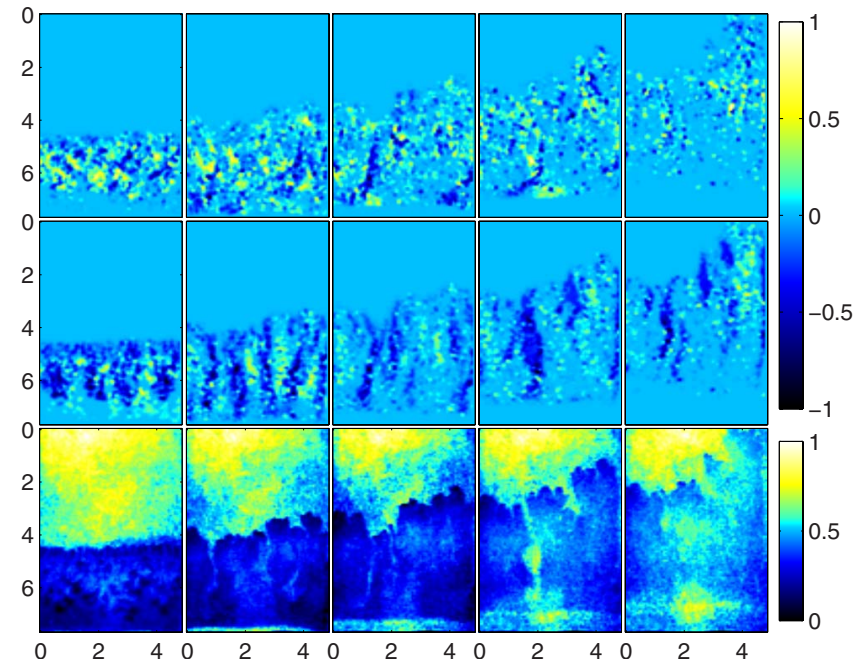

(b)

FIG. 12. (Color online) The three rows in figure (a) and (b) show the time evolution of the simulations (a) and experiments (b) with water/glycerol from left to right at times $t=0.8 \mathrm{~s}, t=4.6 \mathrm{~s}, t$ $=8.4 \mathrm{~s}, t=12.2 \mathrm{~s}$, and $t=16.0 \mathrm{~s}$. From top to bottom the rows illustrate the $u_{x} / \max \left(\left|u_{x}\right|\right)$ component and the $u_{y} / \max \left(\left|u_{y}\right|\right)$ component of the grain velocity vector field and the bottom row shows the density field $\rho_{d} / \max \left(\rho_{d}\right)$. All quantities have been normalized by its maximum absolute value.

simulation directly accessible whereas for the experiments we estimated the density field from the pixel gray values in the pictures. This provides a good estimate for air where a large contrast between bubbles of low particle density and fingers of high particle density exists. However, for water/ glycerol the particle density is more smeared out and the gray value of the pixels is a less good estimate of the density. In this case of the experiment of water/glycerol we will focus on the velocity data. All the quantities in the Figs. 11 and 12 have been normalized to one simply by dividing by the maximum absolute value. For air as well as for water/ glycerol the lower part of the cell is filled with sediments. On top of the sediments appears a second zone with moving particles. In this zone fingers of high particle density are 
falling down. Even further toward the top of the cell particles are still compacted and hardly moving. But in these two sequences a major difference can also be observed. In the case of air it appears that most of the downward-falling particles are part of fingers moving from the top to the bottom of the cell. In this case of air we have clearly defined bubbles of low particle density, that have a large density contrast to the fingers of high particle density. For water/glycerol this is not the case and this strong contrast does not appear. Here the density is more smeared out and the areas between the downward-falling fingers are filled with particles moving upward together with the fluid making the downward-falling fingers less visible.

\section{Velocity field histograms}

To compare the experiments with the simulations and to quantify our observations we calculate the velocity field histograms of the $x$ and $y$ component of the velocity field $u_{x}$ and $u_{y}$ divided by the average absolute value of the velocity $u_{a}$ as defined in Eq. (8). This compensates, that the camera records better the particles close to the glass walls. The result of the corresponding histograms for the sequence of pictures in the air case displayed in Fig. 11 are shown in Fig. 13 and for the water/glycerol case from Fig. 12 the corresponding histograms are shown in Fig. 14. In the histograms it was averaged over three experiments and three simulations resulting in the error bars.

We plotted all the graphs in a semi log plot and good agreement between the simulations and the experiments can be observed. Looking first at the similarities between the case of water/glycerol and air, we find that the histograms for the $y$ component of the velocity field $u_{y}$ are not symmetric showing that there are more areas where particles are moving down than up. In the contrast to the histograms of the $y$ component the histograms of the $x$ component of the velocity field $u_{x}$ are symmetric and following an exponential distribution.

Apart from the similarities in the histograms two differences of the histograms in the case of air and water/glycerol give reason why the observed mixing behavior is so different as shown in Figs. 9 and 10. First the $x$ - and $y$-velocity component histograms for the case of water/glycerol are more wide distributed compared to the case of air. Second if we compare the positive branch of the $y$-velocity component histograms we can see that in the case of air we have much less particles moving upward than in the case of water/glycerol. All these points show that a better mixing of the beads in the case of water/glycerol is observed.

In Fig. 13 where the cell is filled with air the positive branch of the $y$ component of the velocity field shows a wider distribution in the case of simulations than in the case of the experiments. A possible reason for this is that we do not take friction between the particles into account. This causes in the simulations that sediments in some regions were moving upward while for the experiments the sediments were less mobile due to the friction between the particles. It is also possible to see, that the difference between the simulations and the experiments of this positive branch
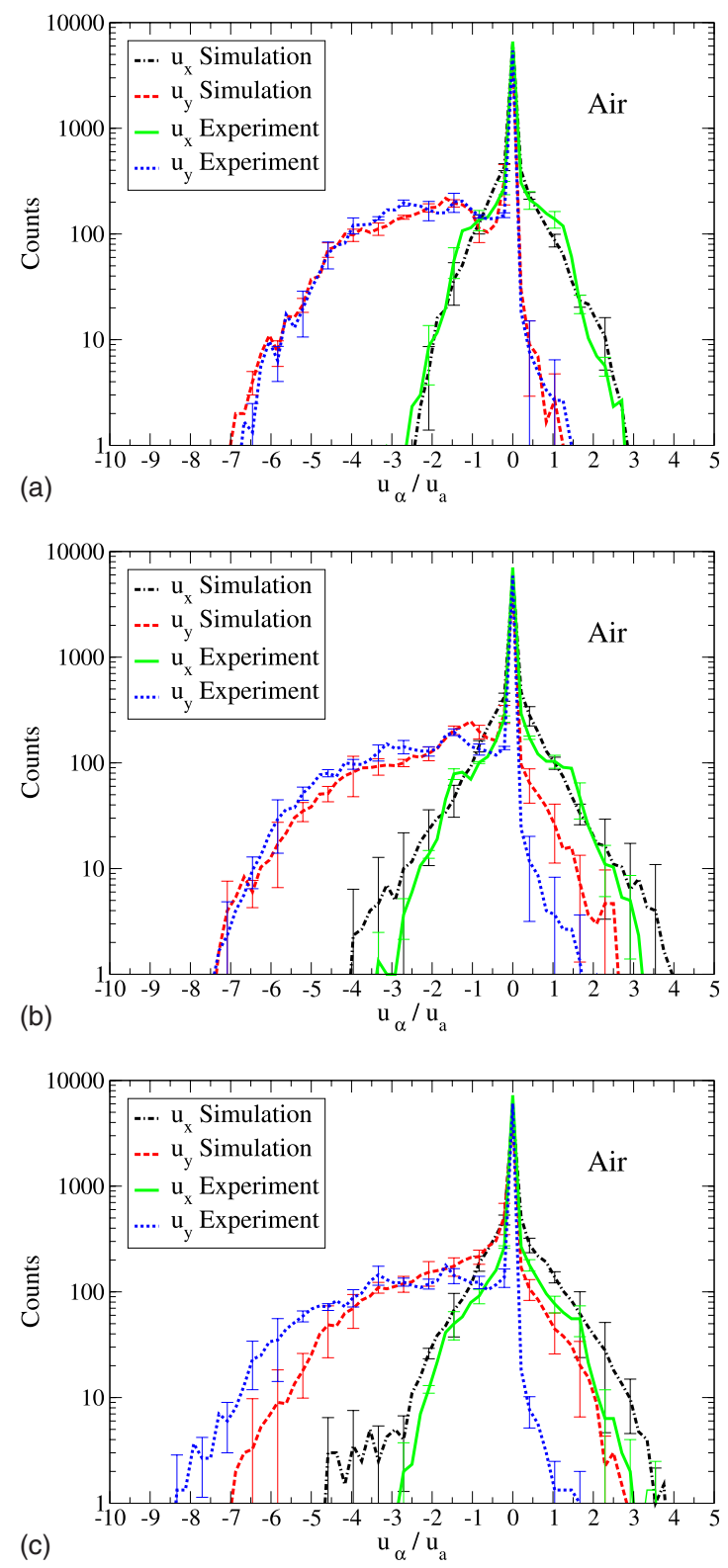

FIG. 13. (Color online) Histograms of the $x$ and $y$ component $\alpha$ of the velocity field after $0.2,0.3$, and $0.5 \mathrm{~s}$ from top to bottom in the case of air. The histograms show good agreement between simulations and experiments.

of the $y$ component of the velocity field is getting larger with time, as more sediments have accumulated.

\section{Autocorrelations}

A way to quantify the size of the dynamic patterns is to calculate the autocorrelation function in $2 \mathrm{D} C_{\alpha}(\mathbf{d})$ where $\alpha$ $=x$, or $y$ the $x$, or $y$ component of the velocity field $\mathbf{u}(i, j)$,

$$
C_{\alpha}(\mathbf{d})=\frac{\sum_{\mathbf{r}}\left\{\left[u_{\alpha}(\mathbf{r})-\left\langle u_{\alpha}\right\rangle\right]\left[u_{\alpha}(\mathbf{r}+\mathbf{d})-\left\langle u_{\alpha}\right\rangle\right]\right\}}{\sum_{\mathbf{r}}\left[u_{\alpha}(\mathbf{r})-\left\langle u_{\alpha}\right\rangle\right]^{2}} .
$$

In this equation the autocorrelation function is normalized by the standard deviation. 

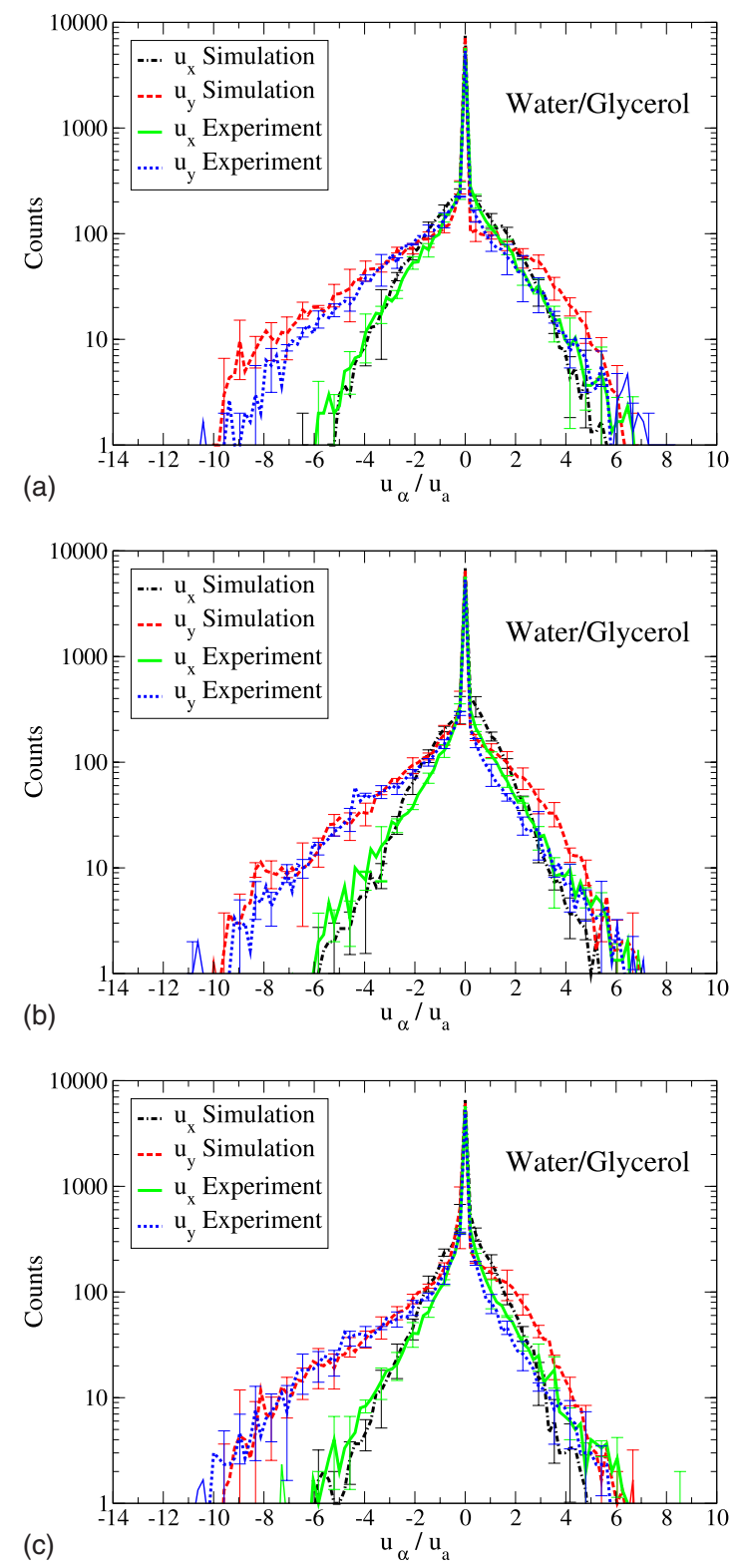

FIG. 14. (Color online) Histograms of the $x$ and $y$ component $\alpha$ of the velocity field after 6,10 , and $15 \mathrm{~s}$ from top to bottom in the case of water/glycerol. The histograms show good agreement between simulations and experiments.

The autocorrelation in 2D is shown in Fig. 15 for air and water/glycerol for corresponding time steps to Figs. 11 and 12. The figures in Fig. 15 demonstrate 2D autocorrelation functions of simulations in part (a) and (c) and of experiments in part (b) (d). The first row of these plots is the 2D autocorrelation function of the $u_{x}$ component of the velocity field and the second row the $u_{y}$ component of the velocity field with time progressing in equal steps from left to right.

In both cases the autocorrelation of the $u_{y}$ component shows periodic vertical lines of stronger correlation directed along the $y$ axis in contrast to the autocorrelation of the $u_{x}$ component of the velocity field which shows equal distribution along the $x$ and the $y$ axis.

The difference between the example of air as a compressible and water/glycerol as an incompressible fluid are more pronounced in the figures for the $u_{y}$ component of the velocity field. In these figures we can observe, that in the case of water/glycerol the periodic vertical lines of stronger correlation are thinner in $x$ direction than in the case of air where these lines take an elliptical shape to the end of the experiments and the simulations.

With time progressing we observe that the frequency of these lines decreases in both cases. This reflects the coarsening process of the structures which we further investigate in Sec. IV. The plots show, that the simulations and the experiments lead to very similar behavior in terms of the autocorrelation field.

\section{Mean wave number}

The temporal evolution of the periodic structures can be measured by performing a Fourier analysis of both components of the velocity field $\mathbf{u}$ and the density field $\rho_{d}$. Since the density field is not accessible directly in the experiments we will use the pixel gray values instead. This approximation is a good estimate in the case of air where we have a large density contrast between the empty bubbles and the downward-falling fingers. However this approximation is a less good estimate in the case of the experiment with water/ glycerol in Fig. 16(d), where the density of the particles is more smeared out and the pixel gray value does not correspond well to the density of the particles.

In this case we are forced to resort to the velocity data only and display the average wave number of the density field for completeness. The average wave number is calculated in the following procedure. For each horizontal line $j$ of the velocity field components $u_{\alpha_{j}}-\left\langle u_{\alpha_{j}}\right\rangle$ or the density field minus the average $\rho_{d_{j}}-\left\langle\rho_{d_{j}}\right\rangle$ the power spectra $S_{j}(k)$ is calculated using a Hamming data window. Taking the average of these power spectra results in a single power spectrum $\bar{S}(k)$ as a function of the Fourier modes $k$. From this distribution the average wave number $\langle k\rangle$ and the standard deviation $\sigma_{k}$ can be calculated by

$$
\begin{gathered}
\langle k\rangle=\frac{\sum k \bar{S}(k)}{\sum \bar{S}(k)}, \\
\sigma_{k}=\sqrt{\frac{\sum k^{2} \bar{S}(k)}{\sum \bar{S}(k)}-\langle k\rangle^{2}} .
\end{gathered}
$$

The two measures are evaluated for the whole cell below the upper surface to avoid contributions apart from the sediments and the bulk. Figure 16 shows $\langle k\rangle$ as a function of time for the $x$ - and $y$-velocity field component and the density field for air and water/glycerol. The error bars are the result from averaging over three different experiments and simulations. In the simulations for air and water/glycerol we observe that the wave number decreases during the first quarter of the total time. This coarsening is less pronounced in the experiments because the initial patterns are strongly perturbated by the rotation of the cell and the initial front contained some minor perturbations. After the first quarter a stable average wave number is achieved with air and with water/ 


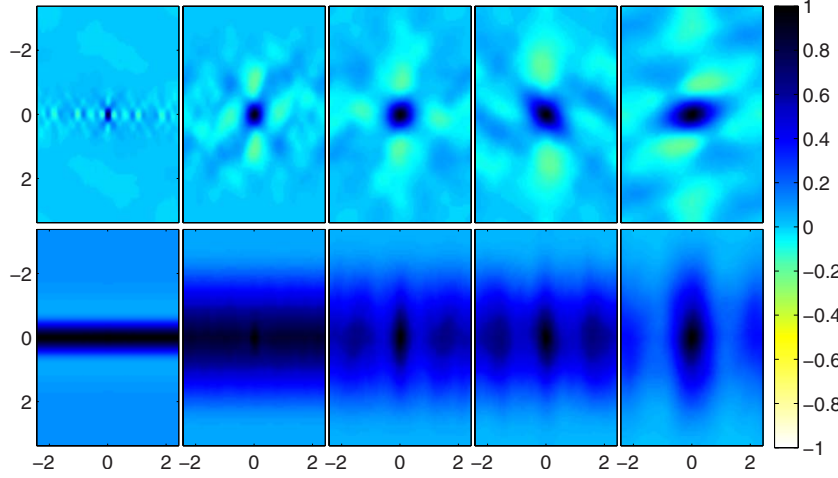

(a)

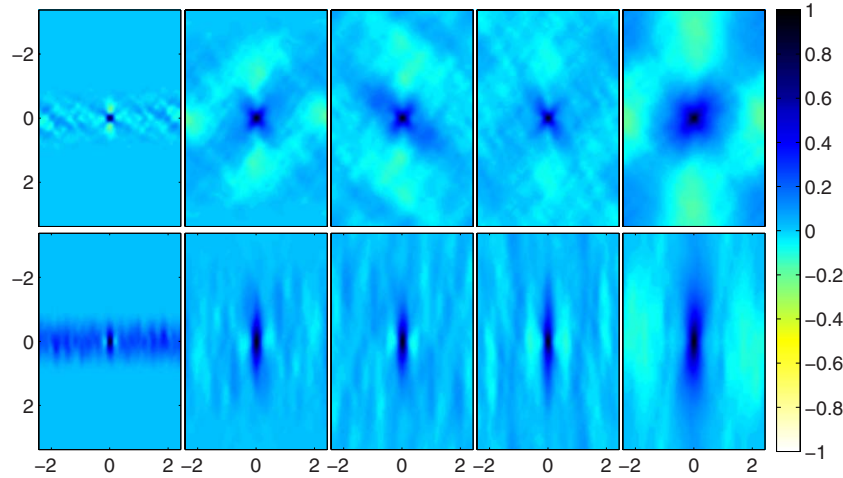

(c)

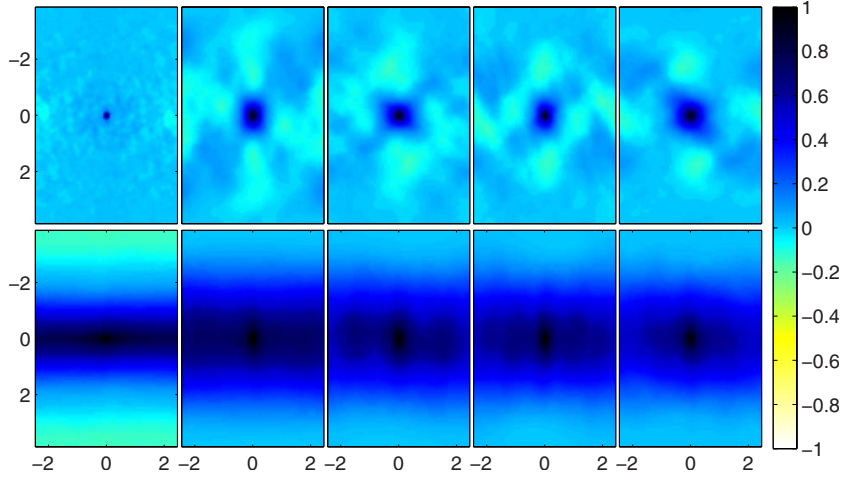

(b)

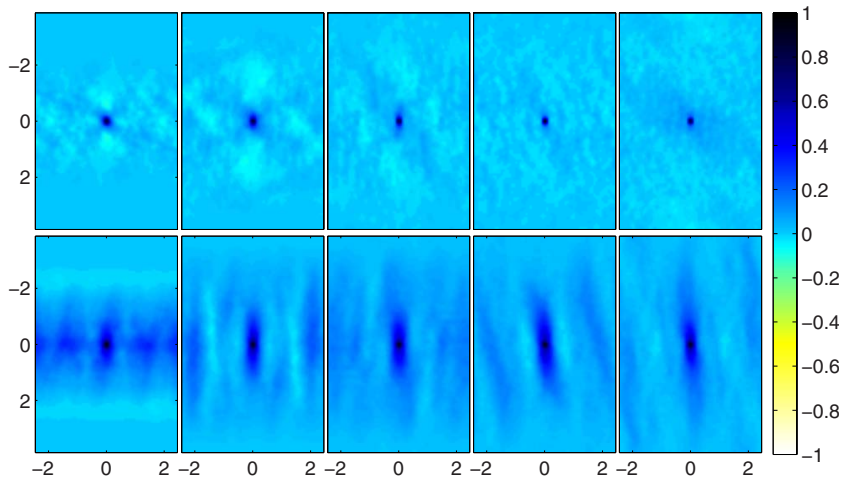

(d)

FIG. 15. (Color online) In the first rows a time sequence of the normalized autocorrelation of the $x$ component of the velocity field $C_{x}(\mathbf{d})$ is shown with $\mathbf{d}=\left(\begin{array}{l}x \\ y\end{array}\right)$. The second rows show a time sequence of the normalized autocorrelation of the $y$ component of the velocity field $C_{y}(\mathbf{d})$. In figure (a) and (b) simulations and experiments with grains and air are presented. Simulations and experiments with grains and water/glycerol are shown in the figures (c) and (d). The time steps from left to right correspond to Figs. 11 and 12.

glycerol. Simulations and experiments result in similar stable average wave number. This average wave number lies around two times higher in the case of water/glycerol than in the case of air.

The figures for the standard deviation of air and water/ glycerol in Fig. 17 show after the first quarter of total time, that the values for water/glycerol are roughly 1.5 times higher than for air. This is the case for both simulations and experiments. The higher values for the standard deviation indicate a wider distribution of the modes in the experiments and simulation with water/glycerol.

\section{PHYSICAL INTERPRETATION OF THE MIXING BEHAVIOR AS FUNCTION OF THE NATURE OF THE INTERSTITIAL FLUID}

To understand the different mixing behavior of the particles in air and water/glycerol we will compare the strength of the particle interaction force through the fluid to the gravitational force. As a first measure we determine if the particles reach terminal velocity. At terminal velocity the force on the particles by the fluid balances the gravitation.

In Fig. 18 the averaged velocity $y$ component multiplied by the fluid viscosity is plotted for the first ten lowest particles until the first particles collide with the base of the cell. Figure 18 shows that the particles in air accelerate continu- ously and do not reach terminal velocity before collision with the base of the cell. On contrary, in water/glycerol the particle reach a characteristic terminal velocity in a very short distance compared to the cell size. In the progress some of the particles in water/glycerol begin to move upward and the average particle velocity in water/glycerol decreases.

The acceleration related to the fluid drag can be calculated in the simulations from the pressure field: $\mathbf{a}_{\mathbf{f}}=\boldsymbol{\nabla} P /\left(\rho_{s} \rho_{m}\right)$. Plotting $\mathbf{a}_{\mathbf{f}}$ in Fig. 19 shows that in water/glycerol the fluid acceleration is comparable and balances the gravitational acceleration of $a_{g}=9.81 \mathrm{~m} / \mathrm{s}^{2}$. In air the fluid forces are increasing as the velocity of the particles increase but never reach the strength of the gravitational acceleration. This also shows that the particles in air collide with the base of the cell before reaching terminal velocity.

As an explanation for the different mixing behavior we shall analyze the fluid velocity field $v_{f}(r, \theta)$ around a falling particle of radius $a$ and velocity $v_{p}$ at position $(r, \theta)$ in polar coordinates in $2 \mathrm{D}$ (because of the plates). Typical solutions of the Stokes equation for a falling sphere or cylinder show that the fluid velocity induced by the motion of this single particle in an otherwise open environment is proportional to the velocity of the particle

$$
v_{f}(r, \theta)=v_{p} f(r, \theta),
$$

where $f$ is a function independent of the viscosity that accounts for the given geometry. In this case the drag force on 

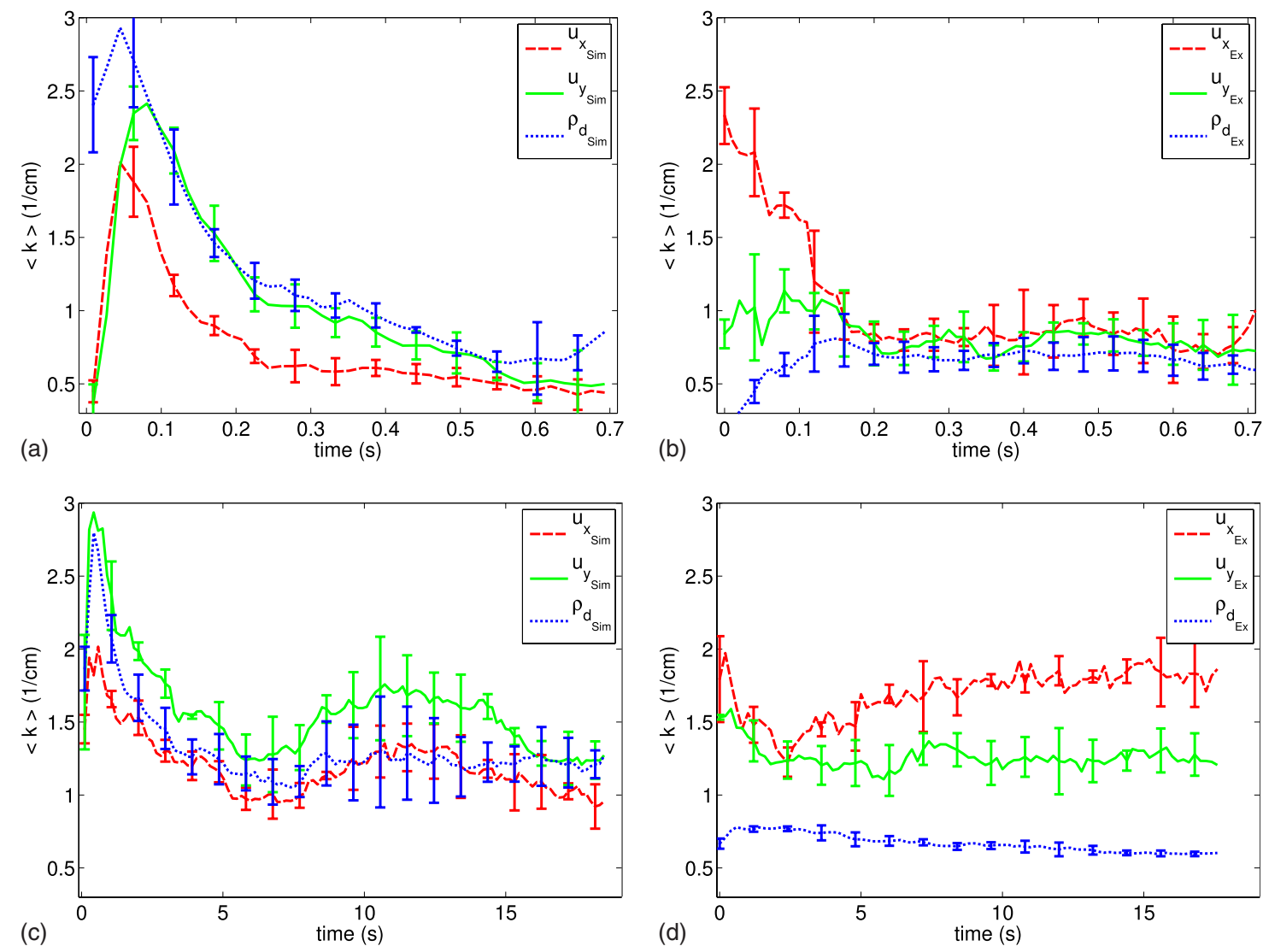

FIG. 16. (Color online) The average wave number $\langle k\rangle$ as a function of time for air and water/glycerol for the velocity field $\mathbf{u}$ and the density field $\rho_{d}$.

the particle is proportional to the viscosity and has the form,

$$
F_{d}=\alpha \mu v_{p},
$$

where $\alpha$ is a constant of the magnitude of the particle size. Given such a drag force the particle will reach a characteristic terminal velocity of

$$
v_{t}=m g /(\alpha \mu) \text {. }
$$

This follows through Newton's second law. The flow field of the falling particle exerts a force on neighboring particles. Using the hydrodynamic doublet approximation [36], this force on a second particle at rest and at position $(r, \theta)$ can be calculated by using Eq. (35) in Eq. (36),

$$
F_{h}=\alpha \mu v_{f}=\alpha \mu v_{p} f(r, \theta)=m g \frac{v_{p}}{v_{t}} f(r, \theta) .
$$

At terminal velocity $v_{p} / v_{t}=1$ and the viscosity dependence cancels out. Once this is reached, the interaction force between the particles has a typical strength independent of the viscosity $\mu$ as shown in Eq. (38). However the particles in air do not reach terminal velocity $v_{p} / v_{t}<1$ and the fluid interaction strength between the particles is weaker than in water/ glycerol. This means that in the dilute region in air the dynamics of the particles are primarily governed by the gravitation force and less by the fluid force. The gravitation is invariant in time and invariant at each position in the cell, which has the effect that the mixing of the particles in air is small. The direction and strength of the fluid force on the other hand depends on time and on the constellation and the velocities of all particles. For each particle trajectory in the cell the fluid force is therefore different. In contrast to the system with air, the fluid force in water/glycerol is larger and comparable to the gravitation because the particles in water/ glycerol reach terminal velocity. The contribution of the fluid force to the total force on the particles in water/glycerol becomes significantly stronger. Hence the direction of the total force in water/glycerol becomes random and can even cause particles to move upward. This causes a better mixing of the particles.

Summarizing, the mixing of the particles is strongly dependent whether terminal velocity is reached or not. At terminal velocity the interaction strength between the particles due to the fluid has the same strength independent of the fluid viscosity. And finally, after terminal velocity is reached the time during the particles are exposed to the fluid interaction forces governs the degree of the mixing.

\section{CONCLUSION}

We have developed a numerical model that describes the granular Rayleigh-Taylor instability for grains mixed either with compressible or incompressible fluids at low Reynolds numbers. In this model we adjusted the fluid compressibility from ideal gas to incompressible behavior. We incorporated 

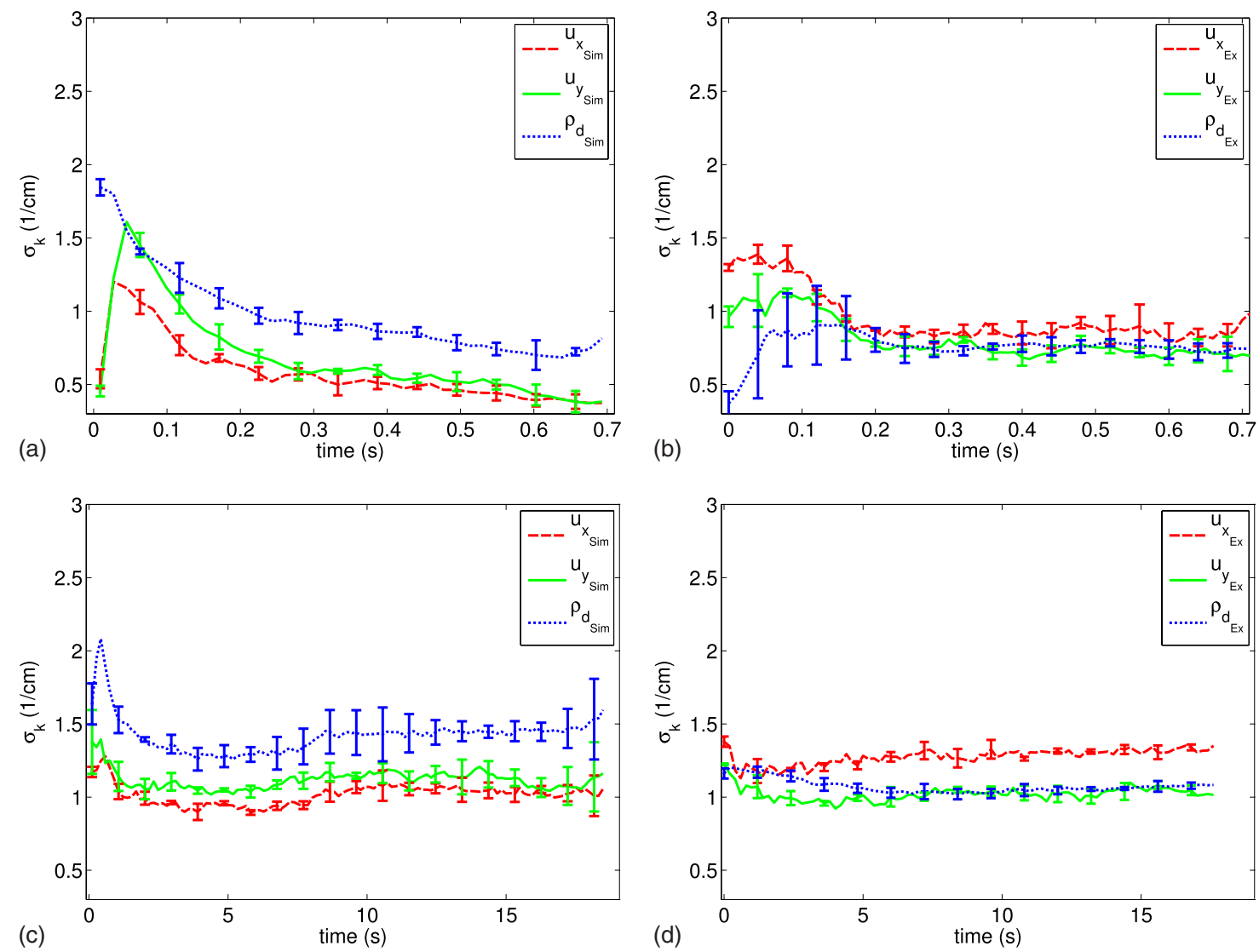

FIG. 17. (Color online) The standard deviation $\sigma_{k}$ as a function of time for air and water/glycerol for the velocity field $\mathbf{u}$ and the density field $\rho_{d}$.

fluid inertia and viscous forces which lead to a hydrodynamic particle-particle coupling and a coupling between the fluid and the confining plates. In addition the model also includes solid friction between the particles and the confining plates. The physical effect of these various terms, their relative importance and the strategies to approximate in simple and computationally efficient ways, have been discussed and evaluated numerically by switching some of these terms on or off and evaluating the effect of the resulting flow. We designed and conducted experiments with compressible and incompressible fluids that where well matched

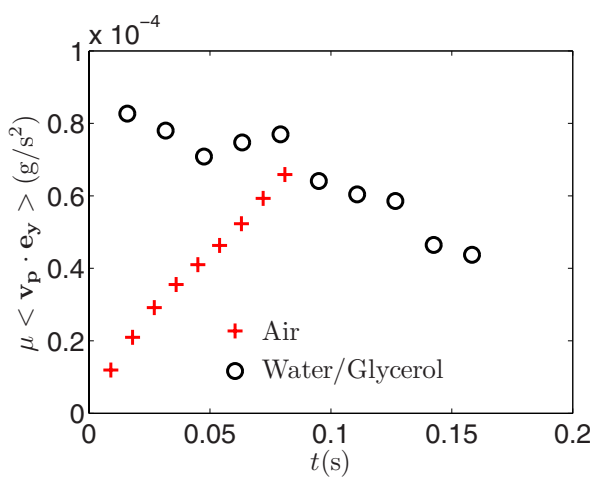

FIG. 18. (Color online) The velocity $y$ component multiplied by the fluid viscosity averaged over the first ten lowest particles in time. quantitatively with our model. From these experiments the velocity field of the particles was extracted by a CIV technique that we improved relative to standard procedures by introducing an additional filtering technique.

The forming patterns and their dynamics were analyzed and compared between the experiments and the simulations. Evaluation of the pictures of the simulation and the pictures of the experiments, velocity field histograms, 2D autocorrelation and the power spectrum of the velocity field showed that our model reproduces well the dynamics of the experiments in both studied cases of water/glycerol and air. Furthermore the analysis of the velocity field and density field resulted in a better understanding of the different mixing

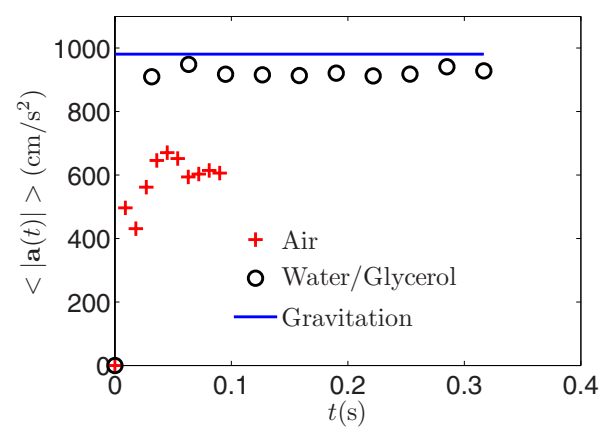

FIG. 19. (Color online) $\mathbf{a}_{\mathbf{f}}=\boldsymbol{\nabla} P /\left(\rho_{m} \rho_{s}\right)$ is the acceleration on the particles due to the fluid in time compared to the gravitational acceleration $\mathbf{a}_{\mathrm{g}}$. The average runs over the first ten lowest particles. 
behavior in the two studied cases as described in chapter I. Two main differences in the histograms and different correlation of the $u_{y}$ component of the velocity field could be identified, which verifies the complete mixing in the case of water/glycerol and the observation of sedimentation patterns in the case of air. Finally in Sec. V we discussed the physical mechanism causing the different mixing behavior as function of the nature of the interstitial fluid

\section{APPENDIX}

In this appendix our model shall be compared to the work of $[23,24]$ where the Navier-Stokes equation is solved directly. In contrast to the work on fluidized beds of $[23,24]$ in our model we will only consider the dominating terms of the Navier-Stokes Eq. (A1) as demonstrated in the following. We will show that this approach also leads to the fluid Eqs. (14) and (21) derived in Sec. III A.

The Navier-Stokes equation for incompressible or lowly compressible fluids in the regime of low Reynolds numbers states,

$$
\partial_{t}\left(\rho_{f} \mathbf{v}_{\mathbf{f}}\right)=-\nabla P+\nabla \cdot T .
$$

In Eq. (A1) the term $\nabla \cdot T=\mu \nabla^{2} \mathbf{v}_{\mathbf{f}}$ accounts for the stress induced by the curvature of the fluid flow profile. It can be separated into a contribution from the fluid flow curvature at small scales, between the particles: $(\boldsymbol{\nabla} \cdot T)_{S}=\mu \nabla_{S}^{2} \mathbf{v}_{\mathbf{f}}$ and a contribution from the fluid flow curvature on a scale larger than the particles: $(\boldsymbol{\nabla} \cdot T)_{l}=\mu \nabla_{l}^{2} \mathbf{v}_{\mathbf{f}}$. The high wave number curvatures $(\nabla \cdot T)_{s}$ of the fluid flow profile between the particles are defined to be separated by a cutoff wave number $K$ from the low wave number curvatures $(\nabla \cdot T)_{l}$. The cutoff wave number $K$ represents the inverse size of the representative volume elements of the Darcy law. With a Fourier decomposition the stress term $T$ can easily be divided into high and low wave number contributions [37],

$$
\begin{aligned}
\mu \nabla^{2} \mathbf{v}_{\mathbf{f}} & =(\boldsymbol{\nabla} \cdot T)_{s}+(\boldsymbol{\nabla} \cdot T)_{l} \\
& =\sum_{k>K}(\boldsymbol{\nabla} \cdot T)_{k} e^{i \mathbf{k r}}+\sum_{k \leq K}(\boldsymbol{\nabla} \cdot T)_{k} e^{i \mathbf{k r} .}
\end{aligned}
$$

To solve Eq. (A1) we consider a discrete square grid of 2.5 particle diameters on which we average the particle velocity and the density. Integrating the force $(\nabla \cdot T)_{S}$ up to this grid scale in the reference frame of the particles leads to Darcy's law in Eq. (12): $\left\langle(\boldsymbol{\nabla} \cdot T)_{s}\right\rangle=\frac{\phi \mu}{\kappa}\left(\mathbf{u}-\mathbf{v}_{\mathbf{f}}\right)$. Thus Eq. (A1) can be rewritten after averaging for small volumes (see [23-26] for details),

$$
\partial_{t}\left(\rho_{f} \phi \mathbf{v}_{\mathbf{f}}\right)=-\phi \nabla P+\frac{\phi^{2} \mu}{\kappa}\left(\mathbf{u}-\mathbf{v}_{\mathbf{f}}\right)+[\boldsymbol{\nabla} \cdot(\phi T)]_{l} .
$$

In this equation the second term on the right-hand side is related to Darcy's law. It is the dominating term together with the gradient of the pressure in the situations we consider. The third term on the right hand side is derived from $(\boldsymbol{\nabla} \cdot T)_{l}$ and accounts for the curvature of the fluid velocity field in a global reference frame. It can be divided into an in-plane and an out of plane contribution,

$$
\begin{aligned}
{[\boldsymbol{\nabla} \cdot(\phi T)]_{l} } & =\left(\boldsymbol{\nabla} \cdot\left\{\mu \phi\left[\left(\boldsymbol{\nabla} \mathbf{v}_{\mathbf{f}}\right)+\left(\boldsymbol{\nabla} \mathbf{v}_{\mathbf{f}}\right)^{T}\right]\right\}\right)_{l} \\
& =\left(\left(\boldsymbol{\nabla}_{\perp}+\boldsymbol{\nabla}_{\|}\right) \cdot\left\{\mu \phi\left[\left(\boldsymbol{\nabla} \mathbf{v}_{\mathbf{f}}\right)+\left(\boldsymbol{\nabla} \mathbf{v}_{\mathbf{f}}\right)^{T}\right]\right\}\right)_{l} .
\end{aligned}
$$

The analysis done in Sec. III C and the measured autocorrelation functions in Sec. IV show that the inertia term $\partial_{t}\left(\rho_{f} \phi \mathbf{v}_{\mathbf{f}}\right)$ in Eq. (A3) is negligible compared to Darcy's law. Furthermore this analysis also shows that the out of plane contribution of the term $\left[\nabla_{\perp}(\phi T)\right]_{l}$ is dominating over the in-plane one. The reason for this is that the fluid flow is correlated in average for $5 \mathrm{~mm}$ in the in-plane direction but only $1 \mathrm{~mm}$ out of plane. Integrating

$$
\left.-\phi \nabla P+\frac{\phi^{2} \mu}{\kappa}\left(\mathbf{u}-\mathbf{v}_{\mathbf{f}}\right)+\left(\boldsymbol{\nabla}_{\perp} \phi T\right)\right]_{l}=0
$$

over the thickness $h$ of the cell we get a shear stress contribution $\sigma_{t}$ from the term $\left.\left(\nabla_{\perp} \phi T\right)\right]_{l}$. With this shear stress Eq. (A5) is equivalent to Eq. (16).

A comparison of this out of plane contribution to the Darcy term was done in Eq. (17)-(20) in Sec. III A. This analysis showed that the out of plane contribution was small compared to the Darcy term. Finally only Darcy's law remains in Eq. (A3) which brings us back to the derivation of the fluid equation in Sec. III A from Eq. (12).
[1] D. Gidaspau, Multiphase Flow and Fluidization (Academic Press, San Diego, 1994).

[2] J. F. Davidson and D. Harrison, Fluidization (Academic Press, New York, 1971).

[3] D. Chehata Gómez, L. Bergougnoux, E. Guazzelli, and J. E. Hinch, Phys. Fluids 20, 023302 (2008).

[4] D. Chehata Gómez, L. Bergougnoux, J. E. Hinch, and E. Guazzelli, Phys. Fluids 19, 098102 (2007).

[5] O. Galland et al., Earth Planet. Sci. Lett. 243, 786 (2006).

[6] D. H. Rothman, J. P. Grotzinger, and P. B. Flemings, J. Sediment Res. 64, 59 (1994).

[7] J. L. Vinningland, Ø. Johnsen, E. G. Flekkøy, R. Toussaint, and K. J. Måløy, Phys. Rev. Lett. 99, 048001 (2007).
[8] J. L. Vinningland, Ø. Johnsen, E. G. Flekkøy, R. Toussaint, and K. J. Måløy, Phys. Rev. E 76, 051306 (2007).

[9] C. Völtz, W. Pesch, and I. Rehberg, Phys. Rev. E 65, 011404 (2001).

[10] C. Völtz, M. Schröter, G. Iori, A. Betat, A. Lange, A. Engel, and I. Rehberg, Phys. Rep. 337, 117 (2000).

[11] A. Lange, M. Schröter, M. A. Scherer, A. Engel, and I. Rehberg, Eur. Phys. J. B 4, 475 (1998).

[12] L. Bergougnoux, S. Ghicini, E. Guazzelli, and J. E. Hinch, Phys. Fluids 15, 1875 (2003).

[13] E. Guazzelli, Phys. Fluids 13, 1537 (2001).

[14] H. Nicolai, B. Herzhaft, E. J. Hinch, L. Oger, and E. Guazzelli, Phys. Fluids 7, 12 (1995). 
[15] H. Nicolai and E. Guazzelli, Phys. Fluids 7, 3 (1995).

[16] Advanced Optical Methods and Applications in Solid Mechan$i c s$, edited by A. Lagarde (Kluwer, Dordrecht, The Netherlands, 2000).

[17] Ø. Johnsen, R. Toussaint, K. J. Måløy, and E. G. Flekkøy, Phys. Rev. E 74, 011301 (2006).

[18] S. McNamara, E. G. Flekkøy, and K. J. Måløy, Phys. Rev. E 61, 4054 (2000).

[19] D.-V. Anghel, M. Strauss, S. McNamara, E. G. Flekkøy and K. J. Måløy, Phys. Rev. E 74, 029906(E) (2006).

[20] M. J. V. Goldschmidt, R. Beetstra, and J. A. M. Kuipers, Powder Technol. 142, 23 (2004).

[21] R. Beetstra, M. A. van der Hoef, and J. A. M. Kuipers, Chem. Eng. Sci. 62, 246 (2007).

[22] M. A. van der Hoef, D. Frenkel, and A. J. C. Ladd, Phys. Rev. Lett. 67, 3459 (1991).

[23] M. A. van der Hoef, M. Ye, M. van Sint Annaland, A. T. Andrews IV, S. Sundaresan, and J. A. M. Kuipers, Adv. Chem. Eng. 31, 65 (2006).

[24] N. G. Deen, M. van Sint Annaland, M. A. van der Hoef, and J. A. M. Kuipers, Chem. Eng. Sci. 62, 28 (2007).

[25] R. M. Iverson, Rev. Geophys. 35, 245 (1997).
[26] R. Jackson, The Dynamics of Fluidized Particles (Cambridge University Press, Cambridge, England, 2000).

[27] P. C. Carman, Trans. Inst. Chem. Engrs. 15, 150 (1937).

[28] H. Darcy, Les Fontaines Publiques de la ville de Dijon (Dalmont, Paris, 1856).

[29] E. Guyon, J. P. Hulin, L. Petit, and C. D. Mitescu, Physical Hydrodynamics (Oxford University Press, Oxford, 2001).

[30] A. Einstein, Ann. Phys. 324, 289 (1906).

[31] H. C. Brinkman, J. Chem. Phys. 20, 571 (1952).

[32] R. Roscoe, Br. J. Appl. Phys. 3, 267 (1952).

[33] E. G. Flekkøy, R. Delgado-Buscalioni, and P. V. Coveney, Phys. Rev. E 72, 026703 (2005).

[34] Behaviour of Granular Materials, edited by B. Cambou (Springer Verlag, Wien, NY, 1998).

[35] L. D. Landau and E. M. Lifshitz, Theory of Elasticity (Elsevier Butterworth-Heinemann, Oxford, 2001).

[36] A. Y. Grosberg and A. R. Khokhlov, Statistical Physics of Macromolecules (American Institute of Physics, New York, 1994).

[37] U. Frisch, Turbulence (Cambridge University Press, Cambridge, England, 1995). 